\title{
Effect of contraction ratio upon viscoelastic flow in contractions: The axisymmetric case
}

\author{
Mónica S.N. Oliveira ${ }^{a}$, Paulo J. Oliveira ${ }^{b}$, Fernando T. Pinho ${ }^{c, d}$, Manuel A. Alves ${ }^{a}, *$ \\ ${ }^{a}$ Departamento de Engenharia Química, CEFT, Faculdade de Engenharia da Universidade do Porto, Rua Dr. Roberto Frias, $4200-465$ Porto, Portugal \\ ${ }^{\mathrm{b}}$ Departamento de Engenharia Electromecânica, Unidade de Materiais Têxteis e Papeleiros, Universidade da Beira Interior, 6201-001 Covilhã, Portugal \\ ${ }^{\mathrm{c}}$ Centro de Estudos de Fenómenos de Transporte, Faculdade de Engenharia da Universidade do Porto, 4200-465 Porto, Portugal \\ ${ }^{\mathrm{d}}$ Universidade do Minho, Largo do Paço, 4704-553 Braga, Portugal \\ Received 11 January 2007; received in revised form 10 July 2007; accepted 10 July 2007
}

\begin{abstract}
A comprehensive numerical study of the effects of the contraction ratio upon viscoelastic flow through axisymmetric contractions was carried out. Six contraction ratios were examined $(\mathrm{CR}=2,4,10,20,40$ and 100) using the Oldroyd-B and Phan-Thien-Tanner (PTT) constitutive equations, under creeping-flow conditions and for a wide range of Deborah numbers $(D e)$. The results enabled the construction of vortex pattern maps, with $\mathrm{CR}$ and $D e$ as independent parameters, elucidating the role of these dimensionless groups in controlling vortex growth, vortex type (lip or corner vortices), and pressure-drop characteristics. The extensional parameter of the PTT model was also varied $(\varepsilon=0-0.5)$ and it was found that for small values of $\varepsilon$ the Couette correction is a monotonic decreasing function of $D e$, while for high $\varepsilon$ values it is a monotonic increasing function.

(c) 2007 Elsevier B.V. All rights reserved.
\end{abstract}

Keywords: Axisymmetric contraction; Viscoelastic fluid; Creeping flow; Contraction ratio; PTT model; Oldroyd-B model

\section{Introduction}

The entry-flow is a long-standing problem dating back to the late 1800s [1]. The early works were mainly experimental and were primarily stimulated by the need to construct a capillary rheometer capable of measuring accurately the viscosity of Newtonian fluids [2]. Since then contraction flows have been the subject matter of numerous experimental and numerical works. The first numerical study of this problem, in which the complete equations of motion were solved, was published by Vrentas et al. [3] and concerned the creeping flow of a Newtonian fluid through an axisymmetric contraction.

In spite of the simple geometry, these flows exhibit complex patterns where shear and extensional regions co-exist. Near the walls the flow is shear-dominated while along the centerline it is purely extensional. These flows are amongst the most studied extensionally dominated flows, since they assume particular importance in industrial applications involving viscoelastic

\footnotetext{
* Corresponding author. Fax: +351 225081449.

E-mail addresses: monica.oliveira@fe.up.pt (M.S.N.Oliveira),pjpo@ubi.pt (P.J. Oliveira),fpinho@fe.up.pt, fpinho@dem.uminho.pt (F.T. Pinho), mmalves@fe.up.pt (M.A. Alves).
}

non-Newtonian fluids. Examples worth mentioning are polymer processing applications, such as injection molding, spinning and film blowing [4]. Literature on contraction flows up until the late 1980s have been the subject of detailed reviews by Boger [1] and White et al. [5], in which the complex effects of flow geometry and fluid rheology were addressed. For a comprehensive account of more recent results the reader is referred to the thorough introductions of Alves et al. [6] and Rodd et al. [7]. An overview of the evolution of numerical methods applied to the flow of viscoelastic fluids through contractions can be found in Walters and Webster [8], which includes typical constitutive models used to represent real fluids; for a more complete review on the theme refer to the book by Owens and Phillips [9]. Here we limit ourselves to a description of some important results with direct relevance to the problem at hand.

\subsection{The sudden contraction configurations}

There are three main types of configuration used in sudden contraction research: the axisymmetric, the planar and the three-dimensional contraction of which the square-square contraction is a particular case. In the first, the fluid flows from a capillary of large diameter through a sudden contraction into a 
smaller capillary; in the corresponding planar case, the capillaries are replaced by channels of large aspect ratio (quasi-2D) and the contraction occurs only in one direction, while in the square-square case this happens in two perpendicular directions.

The flow of viscoelastic fluids through sudden contractions, either planar, axisymmetric or square-square, generates complex flow patterns. In general, these flows comprise regions of strong shearing close to the walls and non-homogeneous extension along the centerline upstream and downstream of the contraction [10]. Yet, it has been shown that the fluid behavior associated with entry flows in different geometries can be quite contrasting as a result of geometric and rheological dissimilarities.

Perhaps the most widely studied configuration is the axisymmetric contraction, mainly due to its implications in pipe/duct flow [11]. Most of the early papers are devoted to the experimental study of this flow (e.g. [12-16]; more recent experimental studies can be found in [10,17-20]). Up to the early 1990s, numerical methods were usually unable to accurately predict the steady contraction flow of viscoelastic fluids, and most efforts were thus dedicated to improving the numerical techniques, as documented in the books of Crochet et al. [21] and Owens and Phillips [9].

Studies on the planar geometry were carried out soon after the pioneering investigations on axisymmetric contractions. The planar geometry assumed relevance as optical experimental techniques evolved, such as flow visualization and especially birefringence, and the number of published works on this configuration increased substantially [22-29]. The flow through planar contractions has also received additional attention in numerical investigations [30-39] since this configuration was chosen as a test case for the assessment and improvement of numerical methods in computational rheology [40].

Some of the findings observed for the axisymmetric geometries are replicated in the planar case, such as the formation of a recirculating region upstream of the contraction and the existence of an extra pressure drop associated with the flow of viscoelastic fluids. However, some major differences have been identified as a result of the different strain and strain-rate histories experienced by fluid elements in the two geometries [1]. Walters and Webster [41] found no significant vortex activity for Boger fluids in the 4:1 planar case, in marked contrast to observations in 4.4:1 circular contractions. However, for shearthinning fluids, vortex growth was observed in both planar and axisymmetric geometries. Evans and Walters $[25,26]$ studied the flows of shear-thinning and constant-viscosity elastic fluids and found strong vortex enhancement at all times for the shearthinning fluids, but once more no significant vortex activity was observed for Boger fluids in planar contractions. Rothstein and McKinley [10] found that in the axisymmetric case, the size of the salient corner vortex formed was smaller than in the planar case. They attributed this outcome to the different Hencky strains experienced by the polymer molecules as the flow changes from uniaxial to planar.

Over the years, little attention has been paid to the flow through square-square contractions, most likely because they are significantly more complex, and in numerical terms a "simple" $2 \mathrm{D}$ simulation is clearly inadequate. In many ways, the square-square geometry can be thought of as an intermediate between the axisymmetric and the planar contraction. Geometrically, similarities between the planar and the square-square contractions are easy to picture. On the other hand, in terms of the actual flow and variation of strain-rates, similarities between circular and square-square contractions have been observed in the experimental study of Walters and Rawlinson [42]. In their study, the differences between the flow of Boger fluids in planar and axisymmetric geometries were also seen to occur between planar and square-square contractions. More recently, a few studies have addressed this geometry, both under an experimental [6] and a numerical [43] perspective.

\subsection{Flow patterns}

One of the remarkable flow features of viscoelastic fluids worth emphasizing is the vortex formation and vortex enhancement mechanism upstream of the contraction plane. In general, for axisymmetric contraction flows, strong vortex enhancement is observed both for Boger fluids and for shear-thinning viscoelastic fluids. However, the vortex characteristics and the way vortex enhancement evolves with the Deborah number can be strikingly different, depending on the flow geometry and the fluid rheology. In some cases the vortex forms near the salient corner and increases in strength, growing steadily upstream and radially inward towards the re-entrant corner $[1,18]$. In other cases the salient corner vortex grows in size with the Deborah number, while simultaneously a lip vortex forms near the re-entrant corner; subsequently, the lip vortex grows radially outwards and forces the corner vortex to decrease in both size and intensity until it is completely overtaken by the lip vortex [10].

Understanding the mechanisms underlying the flow transitions (and inherent vortex enhancement evolution) that take place as the Deborah number is varied has been a main driving force behind the experimental work in this area [9]. This evolution was shown to depend greatly on the fluid rheology. Boger et al. [16] and Boger and Binnington [18] investigated the behavior of two different Boger fluids having similar shear properties and yet found quite different vortex dynamics. It was then recognized that extensional properties had to be taken into account, and Boger [1] suggested the primary parameter to be the extensional viscosity. Recent experimental investigations by Rothstein and McKinley [10,20] corroborate the important role of the extensional viscosity on the dynamics of vortex growth and associated enhanced pressure drop. In this context one of the aims of the current paper is to examine under which conditions one pathway of vortex evolution is preferred over the other.

\subsection{Excess pressure drop}

In addition to the kinematics, another essential flow characteristic is the pressure drop resulting from a sudden change in diameter. The total pressure drop is a result of the pressure drop due to the fully developed viscous flow through the tubes plus the excess pressure drop associated with contraction entrance effects. Apart from being crucial for a proper assessment of the pumping power required in ducts $[44,45]$, it yields information 
about the global state of viscoelastic stresses in the flow [10]. In fact, many researchers have attempted to use the excess pressure as a means to estimate the extensional viscosity of viscoelastic fluids [19,46-51].

The extra pressure drop observed experimentally and predicted numerically for the flow of viscoelastic fluids through sudden contractions can reach values much greater than those exhibited by the corresponding Newtonian (or inelastic) fluids with similar shear viscosity at the same flow rates [10,20,52]. This extra pressure drop is usually presented in dimensionless form in terms of a Couette correction. For shear-thinning fluids, the Couette correction is found numerically to attain a minimum at low Deborah numbers and then to increase as the Deborah number is further increased [37,38,53]. Furthermore, contraction ratio and geometry type have an effect on the magnitude and on the onset of this enhanced pressure drop [10]. Experiments with Boger fluids show a significant increase of the Couette correction with the Deborah number, in marked contrast with the numerical predictions using the UCM and the Oldroyd$\mathrm{B}$ models, for which a strong reduction in the extra pressure drop is found, even leading to a pressure recovery due to elastic effects [35,38]. Rothstein and McKinley [10,20] suggested that the excess pressure drop observed in the experiments with Boger fluids resulted from an extra dissipative contribution to the elastic stress due to a stress-conformation hysteresis in the non-homogeneous extensional flow near the contraction plane. Phillips et al. [54] were able to predict numerically, at least qualitatively, a significant pressure drop enhancement using a closed form of the adaptive length scale model of Ghosh et al. [55] in accordance with experimental observations.

\subsection{The contraction ratio}

Ever since the fifth international workshop on numerical methods in non-Newtonian flows [40], when the planar and axisymmetric contraction geometries with a 4:1 contraction ratio were put forth as benchmark problems in computational rheology, special attention has been granted to geometries with this specific contraction ratio. The reasoning behind the choice of this particular value was based on the known results regarding the flow of Newtonian fluids, where all interesting flow features worthy of note take place for contraction ratios beneath $4: 1$ [52]. Even though it has been recognized a posteriori that for non-Newtonian fluids this choice has been misguided, as many interesting flow characteristics are only apparent for contraction ratios beyond the specific ratio of 4:1 [52], most published numerical works are still concerned with the standard case of the $4: 1$ contraction ratio (e.g. [35,36,38,56-59]). Some authors have used different values of the contraction ratio and there are a few, mainly experimental, studies in which variations of the contraction ratio are considered $[1,10,15,17]$. An exception to this state of affairs in numerical investigations is the work of Alves et al. [39], in which a detailed analysis of the effect of the contraction ratio on the flow of a PTT fluid through a planar sudden contraction was investigated. That work focused on describing the flow patterns and quantifying the vortex characteristics as a function of contraction ratio and Deborah number. For high contraction ratios, it was found that flow features in the vicinity of the corner, such as corner-vortex size, and corresponding streamlines, scale with the upstream length scale and with elasticity given by the Deborah number divided by the contraction ratio, while features near the re-entrant corner (vortex intensity and streamlines) scale with the downstream length scale and with elasticity measured by the Deborah number defined in terms of downstream quantities.

These experimental and numerical studies emphasize the importance of investigating different contraction configurations at varying contraction ratios. Thus, the present work extends the previous study of Alves et al. [39], which was for the planar geometry, by performing a comprehensive numerical analysis on the effects of the contraction ratio on the flow patterns and vortex dynamics in axisymmetric sudden contractions. A comprehensive set of numerical simulations was performed, ensuing new numerical results for the axisymmetric geometry with the following contraction ratios: 2, 4, 10, 20, 40 and 100. For the standard 4:1 ratio, the results given here for vortex size and intensity have controlled accuracy and may therefore be considered as benchmark data for the axisymmetric configuration. A survey of literature for this case and comparison of the existing data reveals as much scatter as that observed for the planar case (shown in Fig. 1 of Alves et al. [38]). In addition, the present work goes one step further by examining other flow characteristics (such as excess pressure drop) and by investigating various constitutive models corresponding to Newtonian fluids, viscoelastic shear-thinning fluids (PTT model) and constant-viscosity Boger fluids (Oldroyd-B model).

The paper is organized as follows. In Section 2, we present the general governing equations and outline the numerical method used to solve them. Section 3 describes the flow geometry and the computational meshes used and in Section 4 we present benchmark data for the standard 4:1 contraction geometry. In Section 5, a systematic study of the effects of contraction ratio and Deborah number on the flow patterns, vortex characteristics and pressure drop is carried out, whereas Section 6 deals with the effect of the rheological model. Finally, in Section 7, we assess the suitability of the normal-stress ratio criterion of Rothstein and McKinley [10] to estimate the onset of lip-vortex activity, before ending the paper with the main conclusions.

\section{Equations and solution method}

The flow of a viscoelastic incompressible fluid can be expressed in terms of its governing equations: conservation of mass, conservation of momentum and a constitutive equation for the extra stress tensor $\tau$ as a function of the flow kinematics. The first two equations can be written as:

$\nabla \cdot \mathbf{u}=0$

$\rho\left[\frac{\partial \mathbf{u}}{\partial t}+\nabla \cdot(\mathbf{u u})\right]=-\nabla p+\eta_{\mathrm{s}} \nabla \cdot\left(\nabla \mathbf{u}+\nabla \mathbf{u}^{\mathrm{T}}\right)+\nabla \cdot \boldsymbol{\tau}$

where $\rho$ is the density of the fluid, $t$ the time, $\mathbf{u}$ the velocity vector, $p$ the pressure, $\eta_{\mathrm{s}}$ the solvent viscosity and $\boldsymbol{\tau}$ is the extra stress tensor given by an appropriate constitutive equation. 
Throughout this paper, we will use three different constitutive models: the Newtonian, the Oldroyd-B model and a simplified form of the Phan-Thien-Tanner (PTT) constitutive equation [60]. These three models can be written with the single following equation:

$$
\begin{aligned}
\lambda & {\left[\frac{\partial \boldsymbol{\tau}}{\partial t}+\nabla \cdot(\mathbf{u} \boldsymbol{\tau})\right]+f(\boldsymbol{\tau}) \boldsymbol{\tau} } \\
& =\eta_{\mathrm{p}}\left(\nabla \mathbf{u}+\nabla \mathbf{u}^{\mathrm{T}}\right)+\lambda\left(\boldsymbol{\tau} \cdot \nabla \mathbf{u}+\nabla \mathbf{u}^{\mathrm{T}} \cdot \boldsymbol{\tau}\right)
\end{aligned}
$$

where $\lambda$ is the relaxation time of the fluid and $\eta_{\mathrm{p}}$ is the polymer zero-shear-rate viscosity $\left(\eta_{0}=\eta_{\mathrm{p}}+\eta_{\mathrm{s}}\right.$, where $\eta_{0}$ is the total zeroshear-rate viscosity).

The PTT model exhibits shear-thinning behavior and has been used extensively in numerical studies involving contraction flows (see, e.g. [37-39] and references therein) and was shown to be appropriate for modeling both polymeric solutions and polymer melts [9], although often this may require the use of a multimode version.

The simplified version used here does not include the contribution from the lower-convected part of the full Gordon-Schowalter derivative, and the linear form of the stress function $f(\tau)$ is selected,

$f(\boldsymbol{\tau})=1+\frac{\lambda \varepsilon}{\eta_{\mathrm{p}}} \operatorname{tr}(\boldsymbol{\tau})$,

where $\operatorname{tr}()$ represents the trace operator and $\varepsilon$ is an extensionrelated parameter of the model, which eliminates singularities in extensional viscosity. The limiting case of $\varepsilon=0$ will also be considered, as it represents an Oldroyd-B model, which is often used to simulate the behavior of constant shear-viscosity Boger fluids. Newtonian fluids are recovered by setting $\lambda=0$.

The governing equations, Eqs. (1)-(3), are solved using a finite-volume method with a time-marching pressure-correction algorithm with fully collocated variable arrangement, as detailed in Oliveira et al. [61]. This methodology has been explained thoroughly in previous works $[35,38,39,62]$ and only a brief outline is given here. The governing equations are discretized on a computational mesh, which in the present case is orthogonal and non-uniform. The meshes used to map the computational domain are created from structured blocks, which in turn are organized in control-volumes or cells. The equations are then discretized and transformed into systems of algebraic equations. These relate the values of each dependent variable $(p, \mathbf{u}, \boldsymbol{\tau})$, evaluated at the cell center, to the values in the neighboring control-volume centers. The time-dependent terms in Eqs. (2) and (3) are retained in the discretization so that a steady-state solution is effectively approached by successive time advancement steps. At each time step, the algebraic equations are solved for the dependent variables, until the norm of the residuals of all the equations drops below a convergence tolerance within the range of $10^{-4}$ to $10^{-6}$. In all cases full convergence was checked by monitoring relevant variables, such as vortex size and intensity, during the simulation procedure. Since in the present work we are interested in steady-state calculations, the time derivative is discretized with an implicit first-order Euler scheme. Central differences are used to discretize the diffusive terms, while the

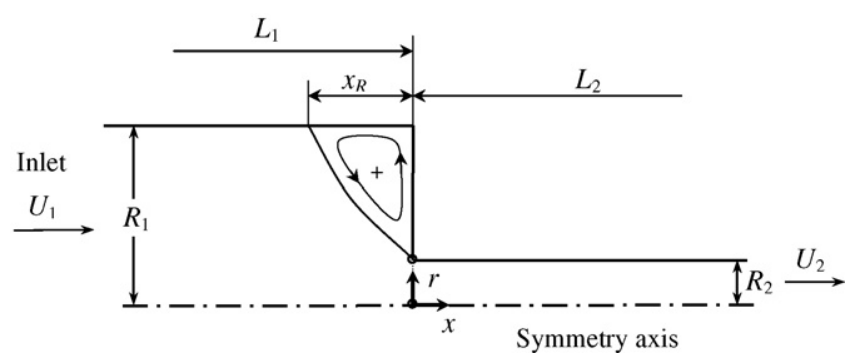

Fig. 1. Schematics of the flow configuration.

CUBISTA high-resolution scheme [62] is employed in the discretization of the advective term of the constitutive equation. The advective term in the momentum equation in neglected since in this work we are interested in truly creeping-flow conditions. The CUBISTA scheme is a bounded version of the QUICK scheme developed by Leonard [63], thus ensuring third-order accuracy in uniform meshes, and was designed to yield a symmetric scheme and to respect Total Variation Diminishing (TVD) restrictions. Alves et al. [39] used it extensively in a study of PTT fluid flow through planar contractions, which is a similar problem to the one we are dealing with in the present paper. For the typical differential constitutive equations found in viscoelastic flows, which are hyperbolic in nature, the CUBISTA scheme was shown to be more appropriate than other highresolution schemes from the point of view of accuracy and iterative-convergence properties [62].

\section{Problem definition and computational meshes}

The geometry of the axisymmetric contraction is depicted in Fig. 1, where $R_{1}$ and $R_{2}$ refer to the radii of the pipes upstream and downstream of the contraction, respectively. The contraction

Table 1

Characteristics of the computational meshes

\begin{tabular}{lrrrrl}
\hline Mesh & $\mathrm{CR}$ & $L_{1} / R_{2}$ & \multicolumn{1}{l}{$L_{2} / R_{2}$} & $\mathrm{NC}$ & $\begin{array}{l}\Delta x_{\min } / R_{2}= \\
\Delta r_{\min } / R_{2}\end{array}$ \\
\hline M1-CR2 & 2 & 2500 & 2500 & 7,732 & 0.020 \\
M1-CR4 & 4 & 2500 & 2500 & 8,980 & 0.020 \\
M3-CR4 & 4 & 2500 & 2500 & 35,920 & 0.010 \\
M1-CR10 & 10 & 2500 & 2500 & 10,420 & 0.020 \\
M1-CR20 & 20 & 2500 & 2500 & 11,956 & 0.020 \\
M1-CR40 & 40 & 2500 & 2500 & 15,796 & 0.020 \\
& 100 & 5000 & 5000 & 23,920 & 0.020 \\
M1-CR100 & 100 & 5000 & 5000 & 95,680 & 0.010 \\
M3-CR100 & & & & & \\
M1-CR4-SHORT & 4 & 40 & 100 & 5,282 & 0.020 \\
M2-CR4-SHORT $^{\mathrm{a}}$ & 4 & 40 & 100 & 10,587 & 0.014 \\
M3-CR4-SHORT $^{\mathrm{a}}$ & 4 & 40 & 100 & 21,128 & 0.010 \\
M4-CR4-SHORT $^{\mathrm{a}}$ & 4 & 40 & 100 & 42,348 & 0.0071 \\
M5-CR4-SHORT $^{\mathrm{a}}$ & 4 & 40 & 100 & 84,512 & 0.0050
\end{tabular}

$\mathrm{CR}$, contraction ratio; $L_{1}$, length of upstream channel; $L_{2}$, length of downstream channel; NC, number of cells; $\Delta x$ and $\Delta r$, cell spacing.

${ }^{\text {a }}$ For $\mathrm{CR}=4$ very refined meshes were used in the benchmark simulations with the Oldroyd-B fluid in order to assess the numerical uncertainty and the true order of convergence. In these simulations smaller inlet and outlet tubes were used. 
ratio between the two tubes, defined as CR $=R_{1} / R_{2}$, is an important geometric parameter and in this study was varied between 2 and 100.

The total number of computational cells was varied depending on the contraction ratio used. Table 1 shows important information on the computational domain and the meshes used, such as the lengths of the upstream and downstream pipes $\left(L_{1}\right.$ and $L_{2}$ ), the total number of cells (NC) and the size of the smallest cell normalized by the radius of the downstream pipe, $\Delta x_{\min } / R_{2}=\Delta r_{\min } / R_{2}$, which is located at the re-entrant corner. All meshes are orthogonal and non-uniform, with the size of

(a)
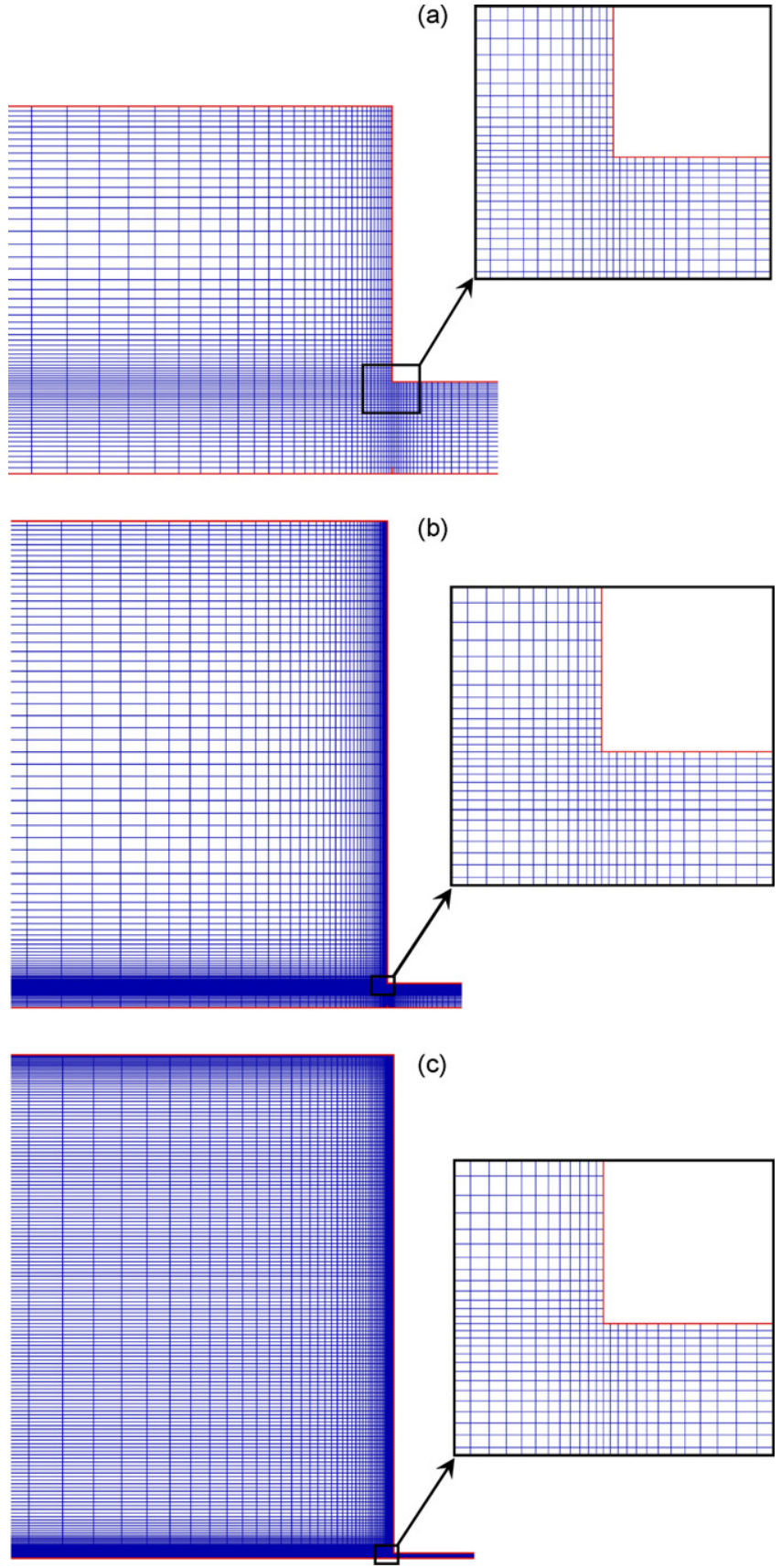

Fig. 2. Zoomed view of the axisymmetric computational meshes with contraction ratios (a) 4:1 (mesh M1-CR4), (b) 20:1 (mesh M1-CR20) and (c) 100:1 (mesh M1-CR100). each cell relating to its neighbors by a geometric progression within each direction, as illustrated in Fig. 2 for contraction ratios of 4, 20 and 100 near the contraction plane. Cell clustering close to the re-entrant corner (shown in the zoomed views of Fig. 2) and along the pipe walls was implemented since the development of thin velocity and stress boundary layers is expected to occur in these regions. The normalized minimum cell spacing near the re-entrant corner is the same for all meshes ( $\left.\Delta x_{\min } / R_{2}=\Delta r_{\min } / R_{2}=0.02\right)$ so that the local variations of the resulting solution fields are not influenced by the mesh resolution. The exception is the benchmark 4:1 test case, for which we have also used more refined meshes in order to assess the accuracy of the numerical results, and the $\mathrm{CR}=100$ case for which a few tests were undertaken using mesh M3-CR100, in order to further assess the numerical accuracy. The tube lengths $L_{1}$ and $L_{2}$ were varied according to the contraction ratio to ensure fully developed flow well upstream of the contraction plane and complete flow redevelopment downstream of the contraction plane. To ensure that this is achieved, we have chosen appropriate pipe lengths based on our previous work for the planar case with a 4:1 contraction ratio [38].

The solvent viscosity ratio $\beta=\eta_{\mathrm{s}} / \eta_{0}$ will for most cases be kept constant at the standard value of $\beta=1 / 9$, while the extensibility parameter of the PTT model will be usually taken as $\varepsilon=0.25$, which is typical of polymer melts or concentrated solutions. PTT fluids exhibit shear-thinning behavior and an extensional viscosity that is strain and strain-rate dependent. The shear and extensional rheometrical properties for the PTT model were presented in Fig. 23 of Ref. [38] and are not repeated here for conciseness. In some cases the $\varepsilon$ parameter will also be varied, ranging from $\varepsilon=0.5$ down to $\varepsilon=0$, representing an Oldroyd-B fluid.

The two other dimensionless groups characterizing the flow are the Deborah and the Reynolds numbers, which are here defined in terms of downstream characteristics,

$D e=\frac{\lambda U_{2}}{R_{2}}$

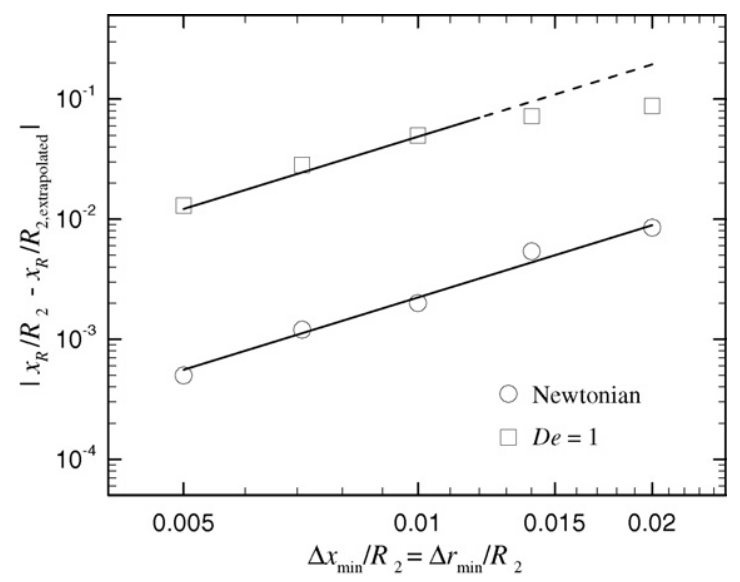

Fig. 3. Estimated error in $x_{\mathrm{R}} / R_{2}$ as a function of mesh size for the Newtonian fluid and for the Oldroyd-B fluid at $D e=1$ (meshes M1-CR4-SHORT to M5CR4-SHORT). 
Table 2

Vortex predictions for $\mathrm{CR}=4$ with Oldroyd-B model $(\beta=1 / 9)$

\begin{tabular}{|c|c|c|c|c|c|c|c|c|}
\hline \multirow[t]{2}{*}{$D e$} & \multicolumn{4}{|l|}{$x_{\mathrm{R}} / R_{2}$} & \multicolumn{4}{|l|}{$\Psi_{\mathrm{R}} \times 10^{3}$} \\
\hline & M2-CR4-SHORT & M4-CR4-SHORT & Extrapolated & Error $^{\mathrm{a}}(\%)$ & M2-CR4-SHORT & M4-CR4-SHORT & Extrapolated & Error $^{\mathrm{a}}(\%)$ \\
\hline 0 & 1.3149 & 1.3107 & $1.3095^{\mathrm{b}}$ & $0.04^{\mathrm{b}}$ & 1.831 & 1.834 & $1.836^{\mathrm{b}}$ & $0.1^{\mathrm{b}}$ \\
\hline 0.5 & 1.602 & 1.598 & 1.597 & 0.1 & 5.70 & 5.45 & 5.37 & 1.5 \\
\hline 1.0 & 1.840 & 1.796 & $1.769^{\mathrm{b}}$ & $0.7^{\mathrm{b}}$ & 12.3 & 10.6 & $9.50^{\mathrm{b}}$ & $5^{\mathrm{b}}$ \\
\hline 1.5 & 2.015 & 1.938 & 1.912 & 1.3 & 20.8 & 16.2 & 14.6 & 11 \\
\hline
\end{tabular}

${ }^{\text {a }}$ Finest mesh data relative to extrapolated value.

${ }^{\mathrm{b}}$ Extrapolated data estimated from calculations in meshes M1-CR4-SHORT up to M5-CR4-SHORT.

$R e=\frac{\rho U_{2} R_{2}}{\eta_{0}}$

where $U_{2}$ is the average cross-sectional velocity in the smaller pipe. The Reynolds number has been set to zero $(R e=0)$, by leaving out the advective term in Eq. (2), to satisfy the creepingflow conditions considered in this work, and the Deborah number was varied from zero (Newtonian flow) up to the maximum value
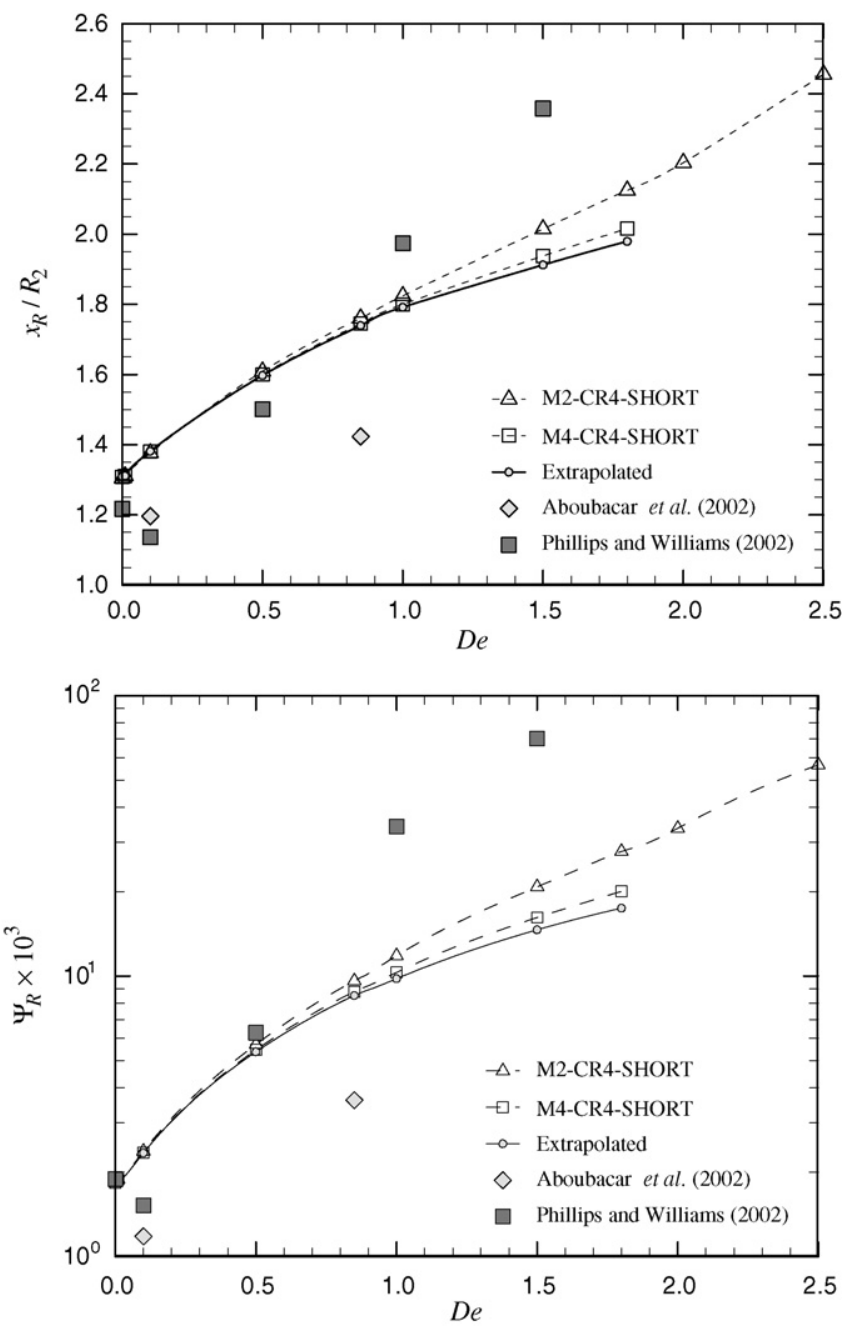

Fig. 4. Variation of the corner-vortex size, $x_{\mathrm{R}} / R_{2}$, and intensity, $\Psi_{\mathrm{R}}$, with the Deborah number for the Oldroyd-B fluid with $\beta=1 / 9$. Comparison with results of Refs. [37] and [64]. where a convergent solution could be obtained. Note that the advective stress term in Eq. (3) is, of course, retained.

\section{Accuracy and benchmark data for $\mathrm{CR}=4$}

For the particular contraction ratio of $4: 1$ a detailed study of convergence with mesh refinement was carried out for the
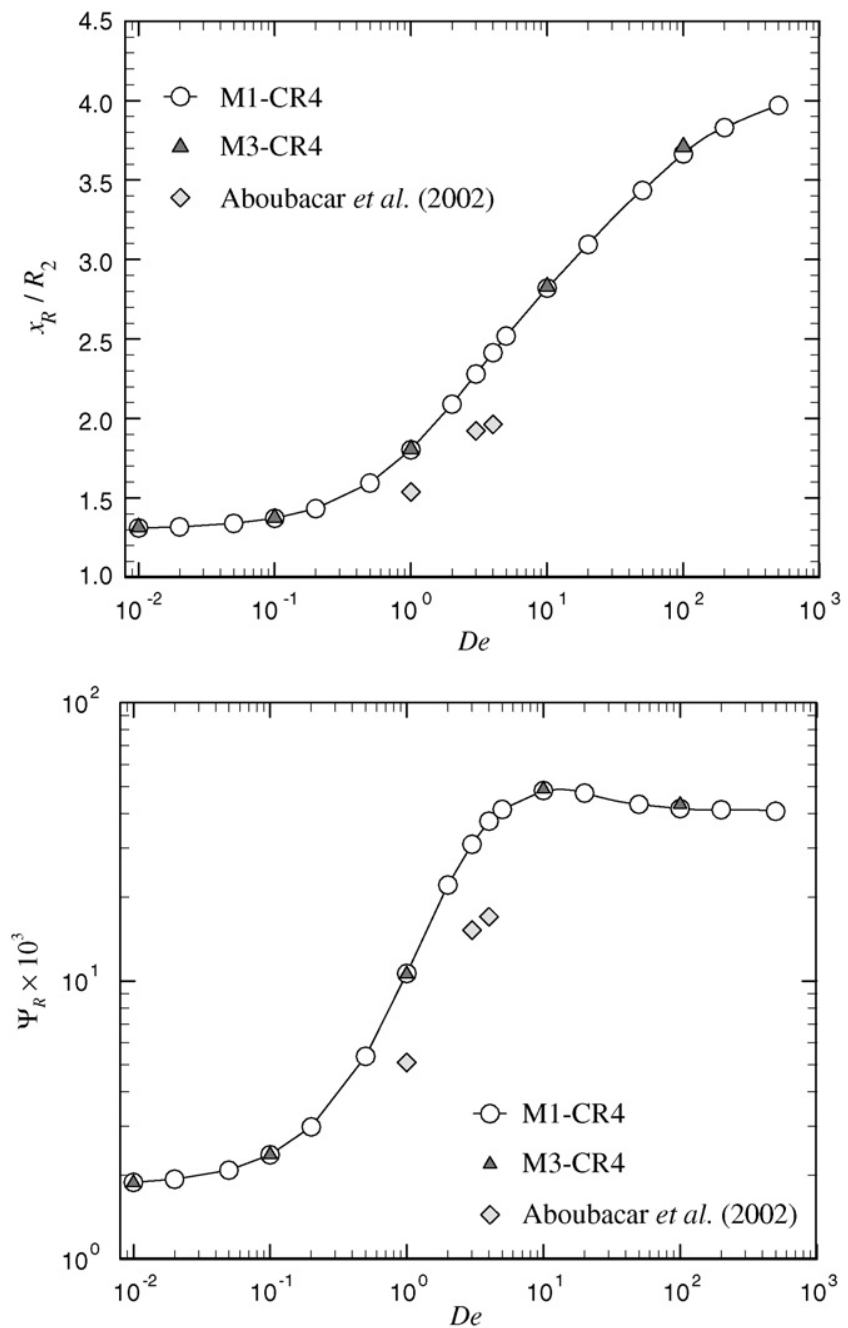

Fig. 5. Variation of the corner-vortex size, $x_{\mathrm{R}} / R_{2}$, and intensity, $\Psi_{\mathrm{R}}$, with the Deborah number for the PTT fluid with $\varepsilon=0.25$ and $\beta=1 / 9$. Comparison with results of Ref. [37]. 
Oldroyd-B fluid ( $\beta=1 / 9$ ) which is useful to quantify the uncertainty of the numerical results. Five meshes were employed having a consistent refinement, with minimum grid spacings of $\left(\Delta x_{\min } / R_{2}=\Delta r_{\min } / R_{2}\right)$ varying from 0.02 down to 0.005 , corresponding to a total number of control volumes ranging from 5282 to 84,512 , as detailed in Table 1 . The use of these meshes allows the estimation of the apparent order of accuracy of the numerical method, and to obtain accurate values using Richardson's extrapolation technique. Table 2 presents the extrapolated results for the size of the corner vortex, $x_{\mathrm{R}} / R_{2}$, and its dimensionless intensity, $\Psi_{\mathrm{R}}$, as a function of the Deborah number. The dimensionless vortex intensity is a measure of the amount of fluid entrapped in the secondary cell and is given by the recirculating flow rate in the corner (or lip) vortex divided by the inlet flow rate into the contraction domain. The estimated accuracy of the numerical results is also included in Table 2, and is quantified by the relative difference between the finest mesh results and the extrapolated values, assuming second-order accuracy. For the Newtonian and $D e=1$ cases we plot in Fig. 3 the estimated absolute error of $x_{\mathrm{R}} / R_{2}$ as a function of the characteristic grid size. Two straight lines with slope of two are included in the figure, thus illustrating the second-order accuracy achieved by the numerical method, at least for the most refined meshes.

In Fig. 4 we present the influence of the Deborah number on the corner-vortex size and intensity for the Oldroyd-B model and compare with results from other authors [37,64]. The accuracy of the numerical results deteriorates as the Deborah number increases, and the maximum attainable $D e$ decreases with mesh refinement, in agreement with previous results obtained for the planar contraction [38].
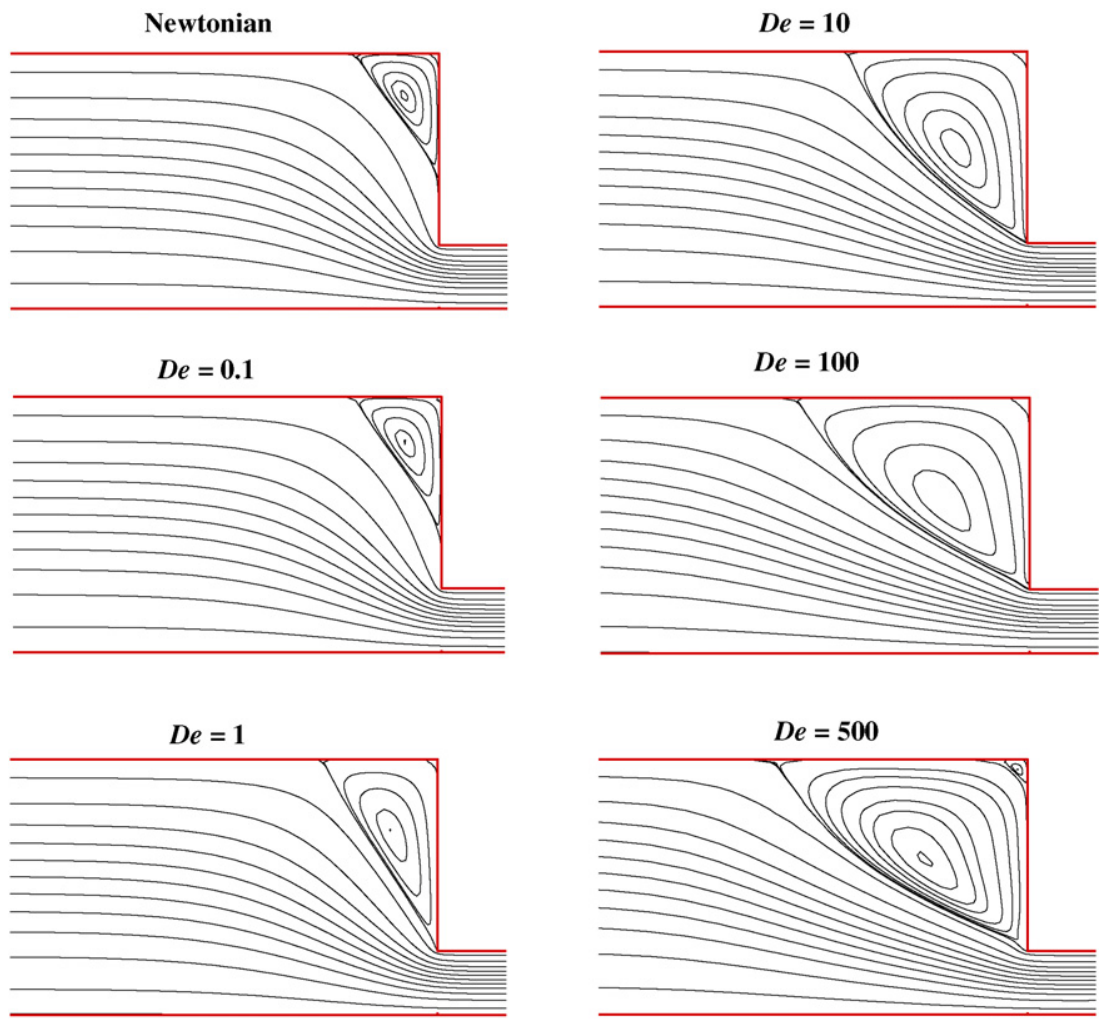

Fig. 6. Streamline patterns for a PTT fluid $(\varepsilon=0.25, \beta=1 / 9)$ in a geometry with a $4: 1$ contraction ratio (mesh M1-CR4). 


\subsection{Flow patterns and streamlines}

The effect of the Deborah number on the flow pattern upstream of the contraction is shown in Figs. 6-8 for contraction ratios of 4, 20 and 100, respectively.

For contraction geometries, vortices form upstream of the contraction and grow in size and strength with elasticity (i.e. Deborah number) independently of the contraction ratio, a behavior which is typical of shear-thinning fluids $[1,25,65]$. The nature of these recirculations, however, depends on the contraction ratio and Deborah number. The examples illustrated in Figs. 6-8 were intentionally selected to illustrate the different vortex trends, enhancements or inhibitions brought about by the Deborah number and contraction ratio.

At low Deborah numbers the corner vortex grows with increasing Deborah number until a lip vortex forms at a critical value of $D e$ which is approximately independent of the contraction ratio provided that the contraction ratio is high (say, $\mathrm{CR}_{\sim} 20$ ). Lip and corner vortices co-exist within an intermediate range of $D e$ that depends on the contraction ratio until eventually the lip vortex grows towards the salient corner and dominates

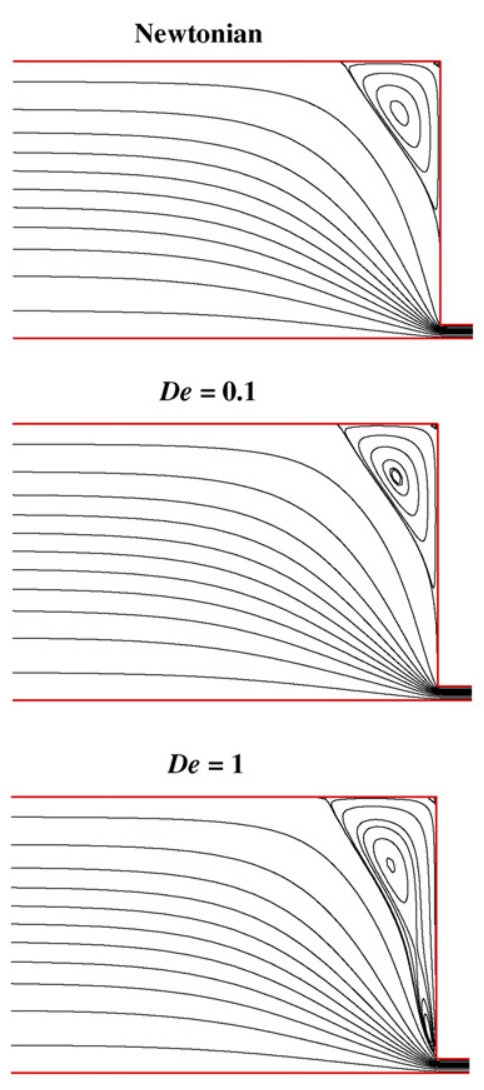

the whole of the recirculating structure. Then, increasing further the Deborah number for the contraction ratios studied here, the large lip vortex continues to grow. As for the planar contraction geometry [39], the transition to a large lip vortex scales with $D e / C R=\lambda U_{2} / R_{1}$, but the critical values for the planar and axisymmetric contractions are different. For high contraction ratios these three stages of vortex evolution are very clear, but this is not the case for the lower contraction ratios tested, at which the co-existence of distinct corner and lip vortices is not observed. This apparent inconsistency can be better understood if it is recognized that for the Deborah number at which the lip vortex was expected to develop, the corner vortex has already grown radially inwards to occupy the whole lateral wall from the salient to the re-entrant corner.

In addition, in each case a weak secondary Moffat vortex is also observed for the whole range of conditions considered in Figs. 6-8. In fact, an infinite series of progressively smaller recirculations should be observed, as predicted by Moffatt [66] for a Newtonian fluid in a corner. Although here we have high Deborah number flows, and therefore the predictions of Moffat are apparently not applicable, the flow in the vicinity of the salient
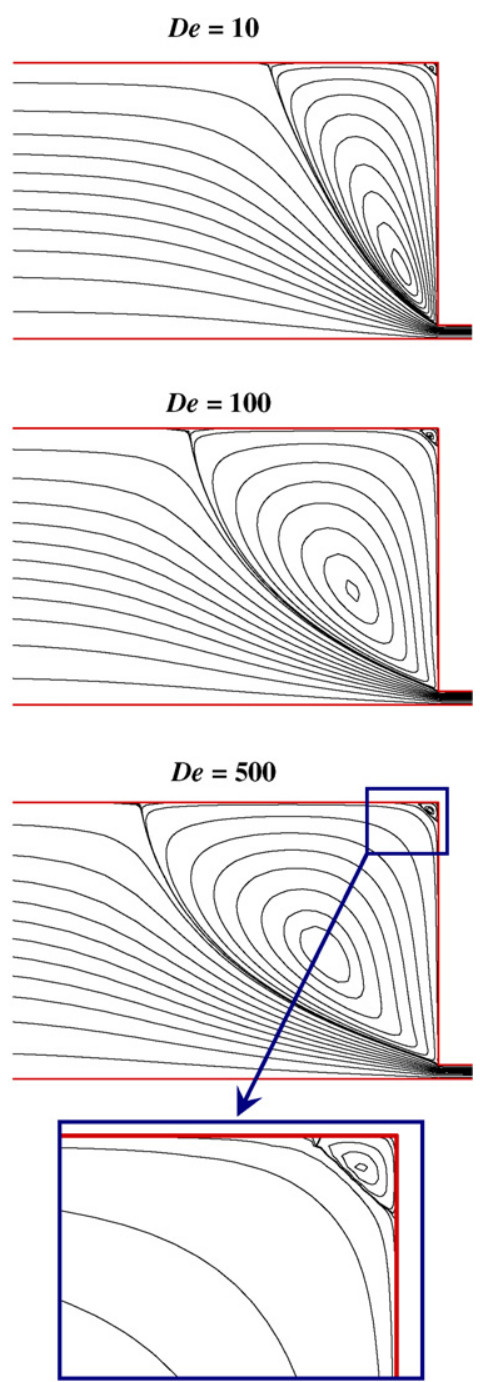

Fig. 7. Streamline patterns for a PTT fluid $(\varepsilon=0.25, \beta=1 / 9)$ in a geometry with a $20: 1$ contraction ratio (mesh M1-CR20). 


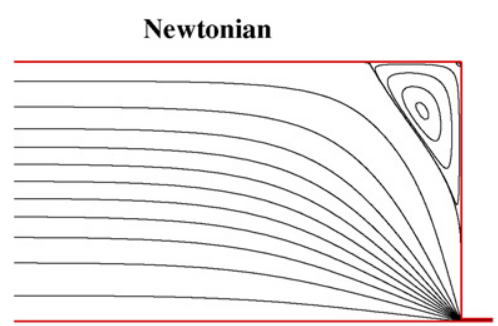

$D e=0.1$

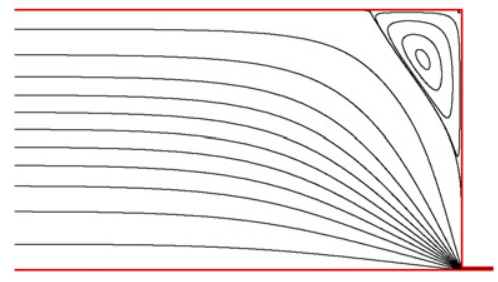

$D e=1$

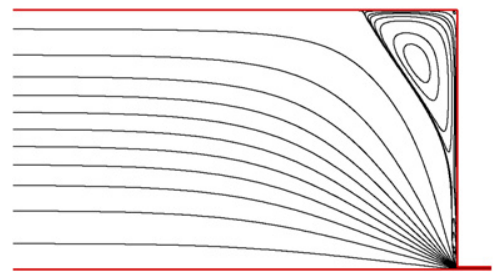

$D e=10$

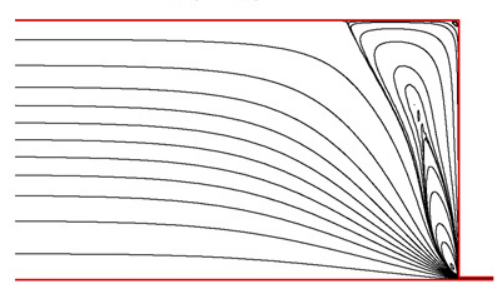

$D e=100$

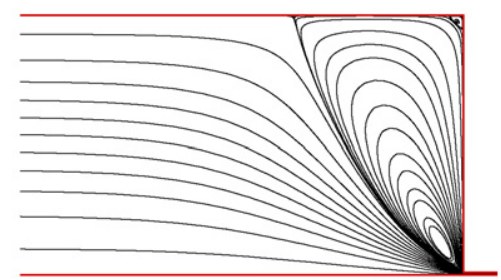

$D e=1000$

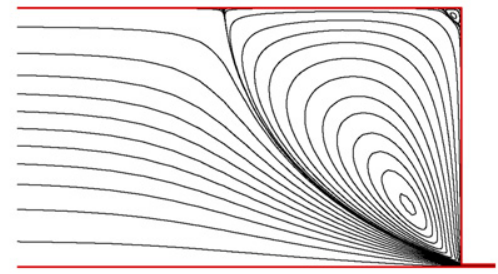

Fig. 8. Streamline patterns for a PTT fluid $(\varepsilon=0.25, \beta=1 / 9)$ in a geometry with a 100:1 contraction ratio (mesh M1-CR100).

corner is locally quasi-Newtonian since the shear rates are low, as compared with the main flow. Due to the finite size of the computational mesh we are unable to predict the third and subsequent recirculations, although the second one is usually well resolved. Generally, this second Moffat vortex is not observed in similar numerical works found in the literature and therefore confirms the good accuracy and resolution of the present results.

In Figs. 9-11 we overlap the streamlines (or $\tau_{x x}$ contour lines) obtained at different $\mathrm{CR}$ and $\mathrm{De}$ to emphasize the correct choice of scaling parameters for local flow characteristics. In Fig. 9, we show the vortices near the re-entrant corner and we superimpose the streamlines for three different contraction ratios $(C R=20$, 40 and 100) maintaining the same downstream duct sizes. This comparison is carried out at the same, low and high, Deborah numbers. There is an almost perfect overlap of the lip vortex near the re-entrant corner, even when a large lip-vortex dominates the structure as observed for $D e=20$ in Fig. 9(b). The collapse of the streamline patterns near the re-entrant corner is clear evidence that the flow field in this region scales with $D e$ for high CR geometries, i.e. the controlling deformation rate is the downstream fully developed shear rate.

To complement this analysis, we show in Fig. 10 the normalstress $\tau_{x x}$ contour plot in the vicinity of the contraction plane. Fig. 10(a) illustrates the effect of CR on $\tau_{x x}$ near the re-entrant corner. In tune with previous observations (Fig. 9), the contour lines in this region are observed to be independent of CR. Although not shown here due to space limitations, this finding is valid for all variables. In Fig. 10(b) we overlap $\tau_{x x}$ contour plots for $\mathrm{CR}=100$ obtained using two meshes with different levels of refinement (M1-CR100 and M3-CR100). It is clear that the contour plot shown is independent of the mesh used except in the region very close to the re-entrant corner, where small differences are observed. These differences are indeed only noticeable at very large magnifications (only about $3 \%$ of the full width of the upstream tube is shown in Fig. 10). Nevertheless, it should be noted that the presence of a stress singularity at the re-entrant corner will repeatedly produce minor deviations in the contour lines even if the mesh is refined further. However, this does not produce a noticeable impact on the flow patterns.

Away from the re-entrant corner the match between the various sets of streamlines is extremely poor, even when we normalize the distances using the radius of the upstream pipe $R_{1}$ (or $\mathrm{CR}$, in non-dimensional terms). If in turn we plot the streamlines corresponding to the same three CR but for three different $D e$ so that the values of Deborah number divided by contraction ratio are identical, e.g. $D e / C R=0.05$, and using the same upstream pipe size, the resulting streamlines near the corner collapse as shown in Fig. 11. Such data collapse demonstrates that the corner vortices scale with the dimensionless group $D e / C R$, i.e. the controlling deformation rate is now essentially the extensional rate of fluid particles moving from the upstream to the downstream pipe, in the same way as observed for planar geometries [39].

Fig. 12 shows a set of vortex pattern maps for the contraction-plane region. These are a result of extensive numerical calculations for various contraction ratios and increasing Deborah numbers. The resulting flow patterns are classified as corner vortex, lip vortex, or as a combination of lip plus cornervortex structures, and are plotted in maps of CR versus $D e$ or De/CR to illustrate the different scaling rules. Fig. 12(a and b) 
(a)

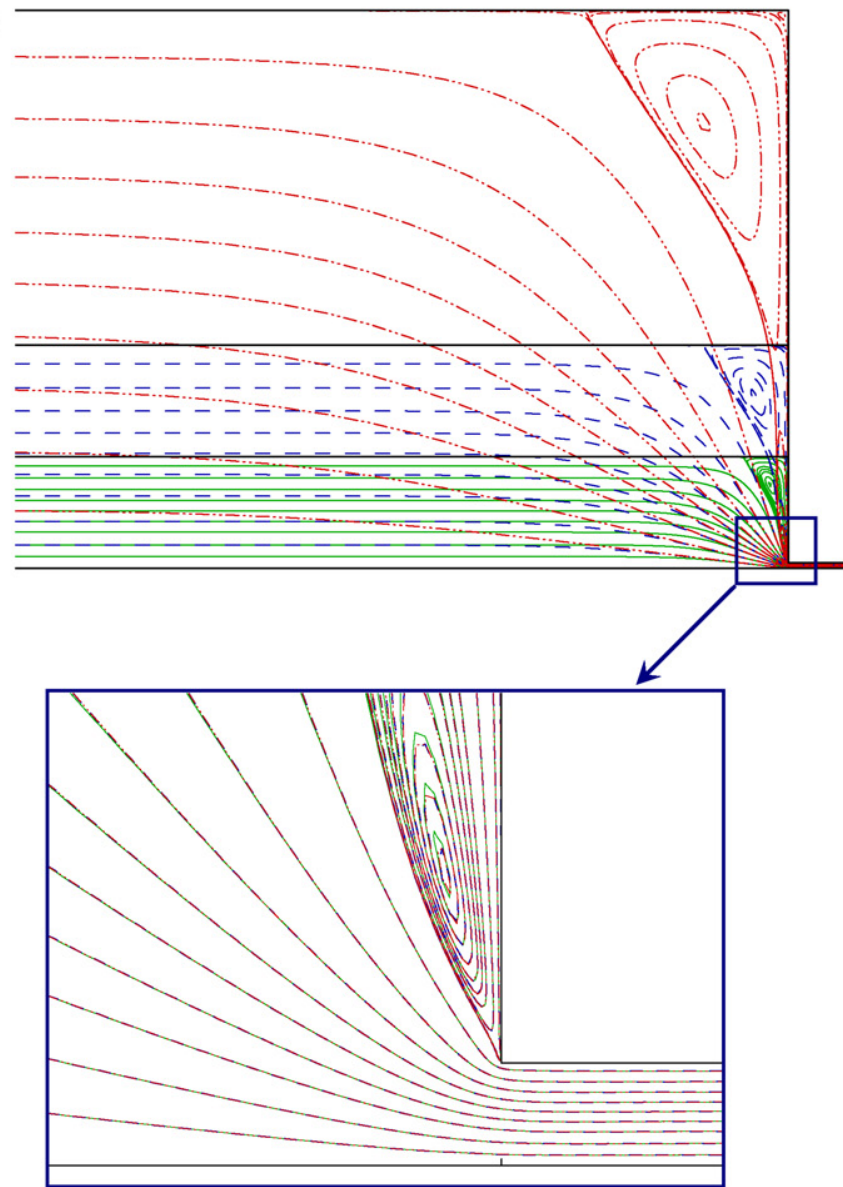

(b)

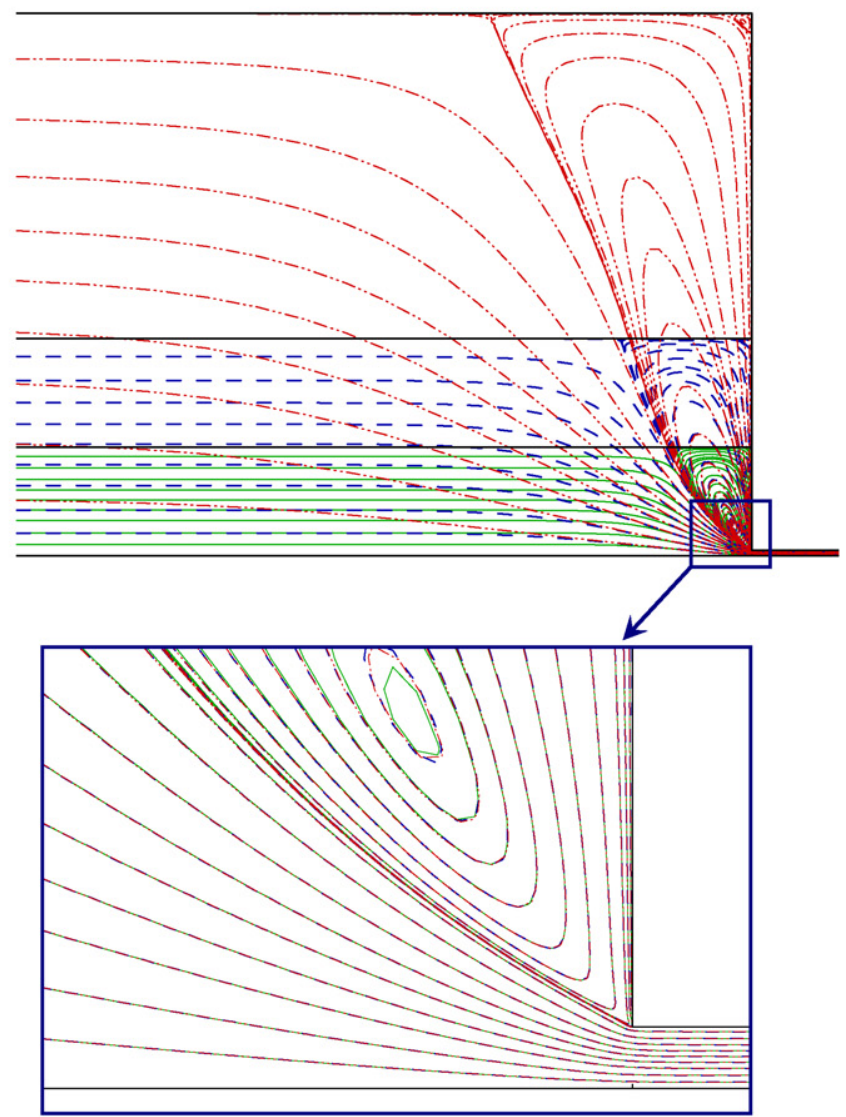

Fig. 9. Comparison of streamlines for a PTT fluid $(\varepsilon=0.25, \beta=1 / 9)$ near the re-entrant corner for three contraction ratios $(\mathrm{CR}=20,40$ and 100$)$ under standard scaling: (a) $D e=1$; (b) $D e=20$.

represent the vortex maps for the axisymmetric contraction and Fig. 12(c and d) depict the equivalent flow maps for a planar contraction based on data from Alves et al. [39]. It is immediately clear that, for contraction ratios greater than 10 , the onset of formation of a lip vortex occurs at a fixed Deborah number, while the point at which the lip vortex engulfs the corner vortex occurs at a constant value of $D e / C R$. Even though the overall structure of the flow field in both axisymmetric and planar contractions is strikingly similar, with the same type of vortices and scaling rules found for both cases, the geometry of the contraction leads to a shift of the critical values of $D e$ and $D e / C R$. The vertical line in Fig. 12(a) for $0.1 \leq D e \leq 0.2$ demonstrates that lip-vortex development is controlled by $D e$. In the axisymmetric contraction, this transition arises at an earlier Deborah number than in the corresponding planar geometry (c.f. Fig. 12(a and c)). The onset of a single merged vortex is dictated by $D e / C R$ as is apparent in the vertical line in Fig. 12(b and d). Likewise, for the axisymmetric case the transition occurs at a lower value of the controlling parameter than that for the planar case.

\subsection{Vortex size and intensity}

In this section, the vortex trends and enhancement mechanisms are analyzed in more detail by quantifying the size and intensity of both corner and lip vortices.
The size of the vortex attached to the salient corner is denoted here by $x_{\mathrm{R}}$ and is measured as the longitudinal length from the contraction plane to the edge of the vortex (along the axial direction) as shown in Fig. 1. The effect of dimensionless number $D e / C R$ on this dimensionless vortex size (normalized with the upstream channel radius $R_{1}$ ) is shown in Fig. 13 for a range of contraction ratios $(2 \leq \mathrm{CR} \leq 100)$. When plotted on a semi$\log$ scale, a sharp increase in the slope of the curve is observed at $D e / \mathrm{CR} \approx 0.1$ compatible with Fig. 12(b). Good agreement between curves for different contraction ratios is observed for $\mathrm{CR} \geq 10$. For smaller contraction ratios the agreement is not as close since the flow in the smaller pipe has a greater influence upon the flow features in the salient corner area. On the contrary, for high $\mathrm{CR}$, the characteristics of the corner vortex are only affected by upstream events and close agreement between different CR is observed.

The variation of the dimensionless corner-vortex intensity, $\Psi_{\mathrm{R}}$, with $D e / \mathrm{CR}$ is plotted in Fig. 14 for the same contraction ratios as above. Again, a reasonable correlation is achieved for $\mathrm{CR} \geq 10$. These data provide further evidence that $D e / \mathrm{CR}$ is the correct scaling parameter for the corner-vortex intensity.

In Fig. 15, the dimensionless lip-vortex intensity, $\Psi_{\mathrm{L}}$, is plotted as a function of the Deborah number for contraction ratios higher than 10 . Here, the lip-vortex intensity is calculated in the 

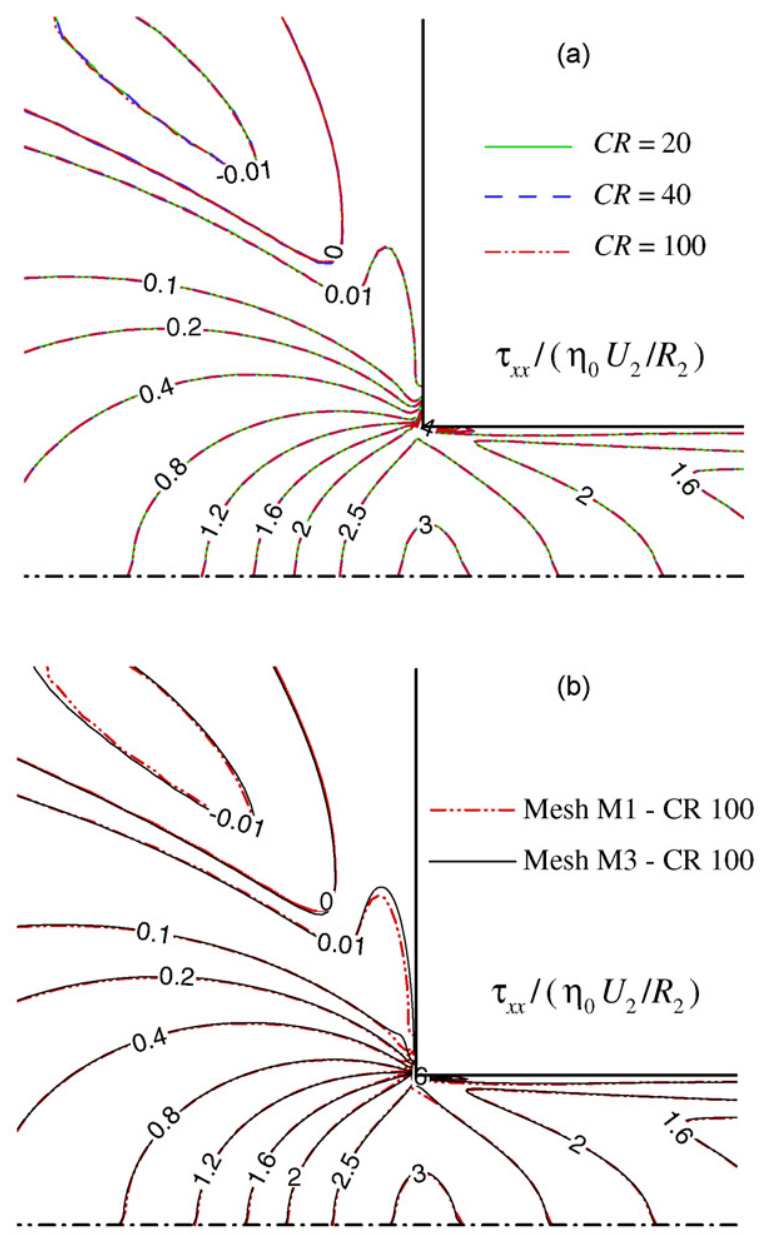

Fig. 10. Contour plots of $\tau_{x x} /\left(\eta_{0} U_{2} / R_{2}\right)$ near the re-entrant corner for the PTT fluid at $D e=20$. (a) Influence of CR (mesh M1) and (b) influence of mesh refinement for $\mathrm{CR}=100$.
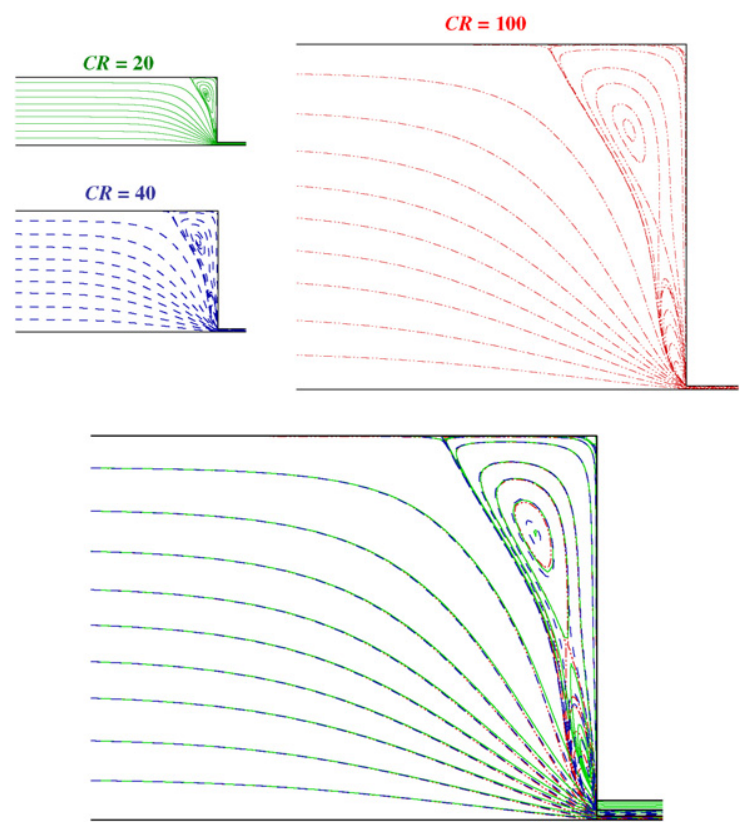

Fig. 11. Comparison of streamlines for a PTT fluid $(\varepsilon=0.25, \beta=1 / 9)$ near the corner after scaling distances with upstream channel height for three contraction ratios $(\mathrm{CR}=20,40$ and 100$)$ under modified scaling $(D e / C R=0.05)$. cases when a lip and a corner vortex exist simultaneously or when the lip vortex merges with the corner vortex. As expected from the previous considerations $\Psi_{\mathrm{L}}$ scales with $D e$, further emphasizing that this is indeed the parameter controlling lipvortex generation and growth.

Incidentally, the maximum $D e$ slightly increases with the contraction ratio as shown also in Fig. 15. A similar result was observed by Alves and Poole [67] in the flow through smooth contractions, where a simple theoretical analysis is presented to explain this finding.

\subsection{Pressure drop}

Associated with vortex enhancement, an extra pressure loss has been experimentally measured in viscoelastic liquid flows through axisymmetric contractions [8]. The total pressure drop $\left(\Delta P_{\text {total }}\right)$ between a location in the large pipe upstream of the contraction and a location in the small pipe downstream of the contraction results from a combination of the pressure drop due to fully developed Poiseuille flow ( $\Delta P_{\text {Poiseuille }}$ ) in each pipe and an extra pressure drop $\left(\Delta P_{\text {ext }}\right)$ caused by the extensional flow in the contraction:

$\Delta P_{\text {total }}=\Delta P_{\text {Poiseuille }}+\Delta P_{\text {ext }}$

This extra pressure loss is usually well above that expected from estimates based on shear-viscosity alone $[8,10]$ and is often made dimensionless using the downstream fully developed wall shear stress $\left(\tau_{\mathrm{w}}\right)$ in the form

$C=\frac{\Delta P_{\mathrm{ext}}}{2 \tau_{\mathrm{w}}}$

where $C$ is termed the Couette correction.

This extra pressure drop is an important flow parameter and during the fifth international workshop on numerical methods in non-Newtonian flow it was proposed as a reference parameter for the benchmark 4:1 contraction flow problem [40].

The evolution of the dimensionless extra pressure drop with the Deborah number is shown in Fig. 16 for various contraction ratios ranging from 10 to 100 . In addition, we have plotted the Couette correction based on Sampson's analytical solution for the Stokes flow of a Newtonian fluid through a circular orifice of zero thickness in an infinite wall and use it to assess the predictions at low $D e$. The Sampson pressure drop is given by [45]:

$\Delta P_{\mathrm{S}}=\frac{3 Q \mu}{R_{2}^{3}}$

where $R_{2}$ is the orifice radius and $\mu$ is the constant shearviscosity. As Sisavath et al. [45] highlight, for a contraction geometry under creeping-flow conditions, the flow is symmetric and as a result the extra pressure drop due to the entrance flow is half the extra pressure drop through a thin orifice. Therefore, we can estimate the Couette correction as $C=\left(\Delta P_{\mathrm{S}} / 2\right) / 2 \tau_{\mathrm{w}}=3 \pi / 16$. We should emphasize that this result is only (approximately) valid for creeping-flow conditions and high contraction ratios. 

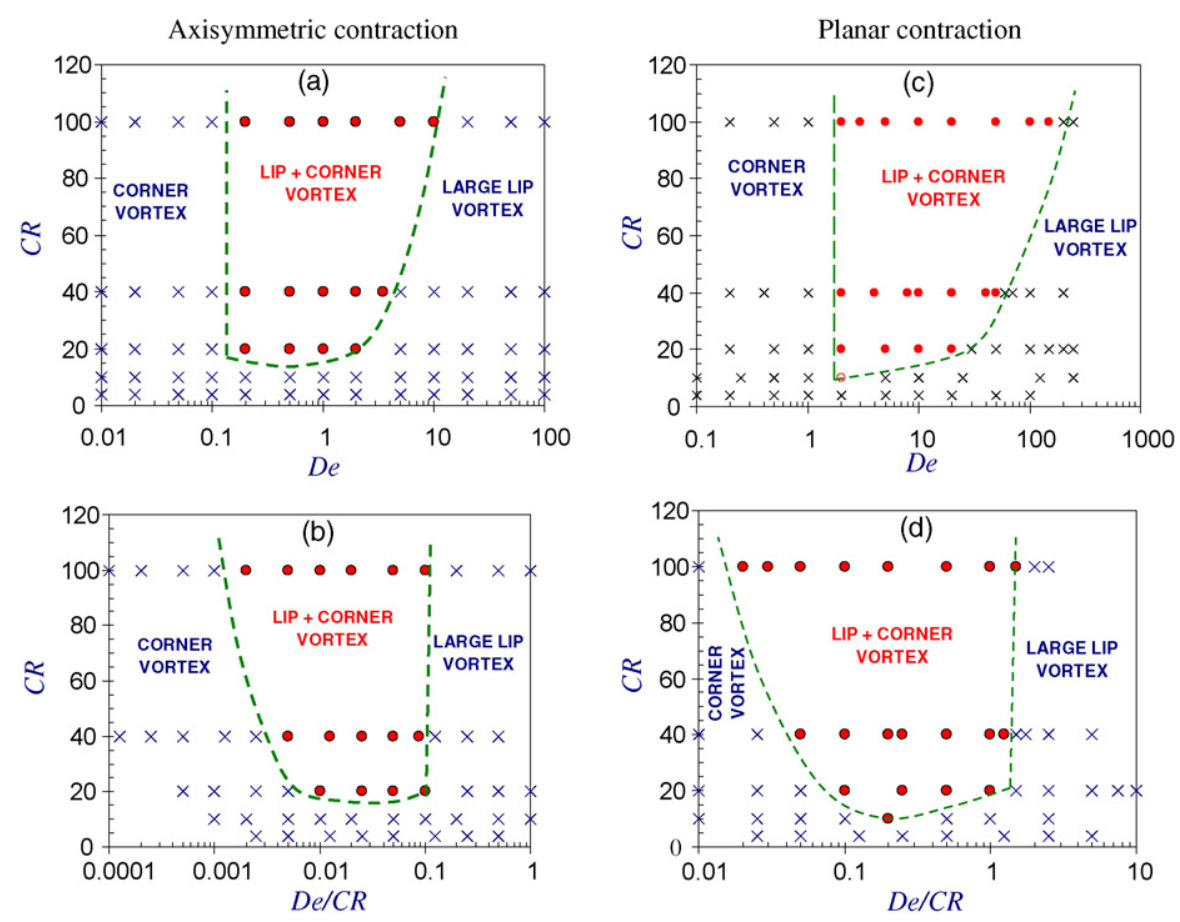

Fig. 12. Vortex pattern maps for the axisymmetric and planar case under: (a and c) standard scaling; (b and d) modified De scaling.

For the smallest $D e$ numbers, the numerically calculated Couette correction approaches asymptotically the value predicted by Sampson's equation, at least for the large contraction ratios used here (we get $C=0.575$ for $\mathrm{CR}=100, D e=0$ ). For lower contraction ratios, consistent with the experimental observations of Rothstein and McKinley [10] and references therein, the assumption of a lateral wall of infinite extent ceases to be a good approximation. As we increase the Deborah number, extensional effects start to play an important part-non-Newtonian behavior is clearly visible as the Couette correction deviates from the Sampson's estimation, increasing substantially for $D e>1$. In any case, for the large contraction ratios, the Couette correction scales with the Deborah number.

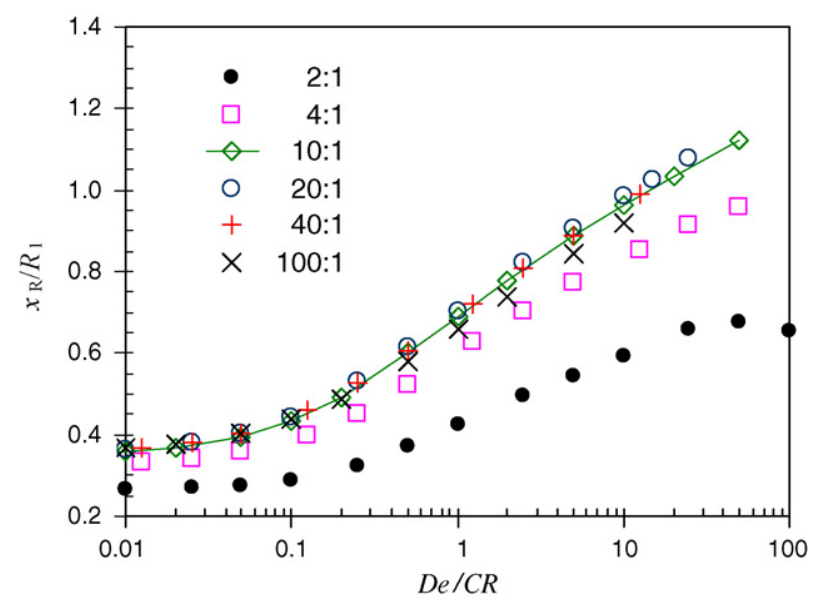

Fig. 13. Variation of corner-vortex size, scaled with upstream tube radius, with $D e /$ CR for a PTT fluid $(\varepsilon=0.25, \beta=1 / 9)$.

\section{Effect of the rheological model}

As described in the Introduction, the behavior of constantviscosity and shear-thinning elastic fluids can be quite distinct, depending on the flow conditions. The aim of this section is to establish a comparison between the flow behavior triggered by the use of different parameters of the rheological models.

We have performed a detailed study of the effect of the $\varepsilon$ parameter of the PTT model on the vortex features and extra pressure drop. The value of $\varepsilon$ was varied from 0.5 to 0 , i.e. down to the limiting case of the Oldroyd-B model. The use of the Oldroyd-B constitutive equation in the simulations presents an additional numerical difficulty, and convergence could not be achieved for high Deborah numbers. As a consequence, for the

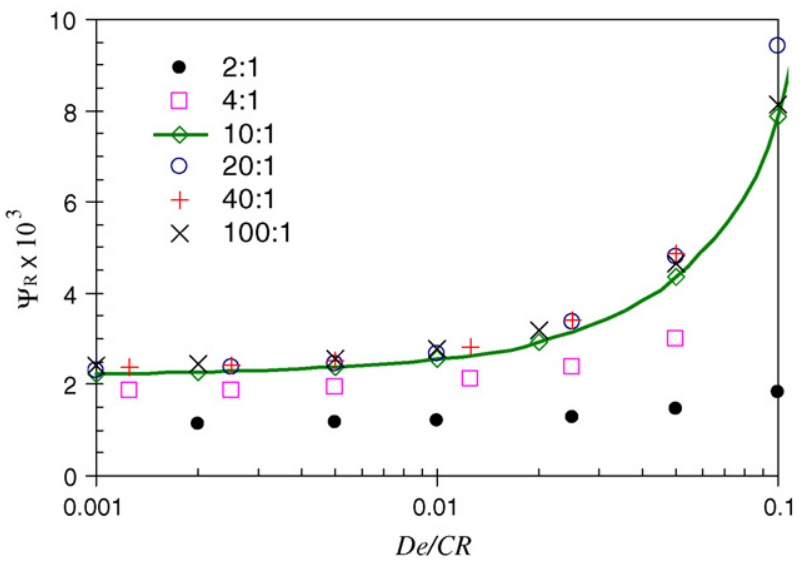

Fig. 14. Variation of corner-vortex intensity with modified De scaling for a PTT fluid $(\varepsilon=0.25, \beta=1 / 9)$. 


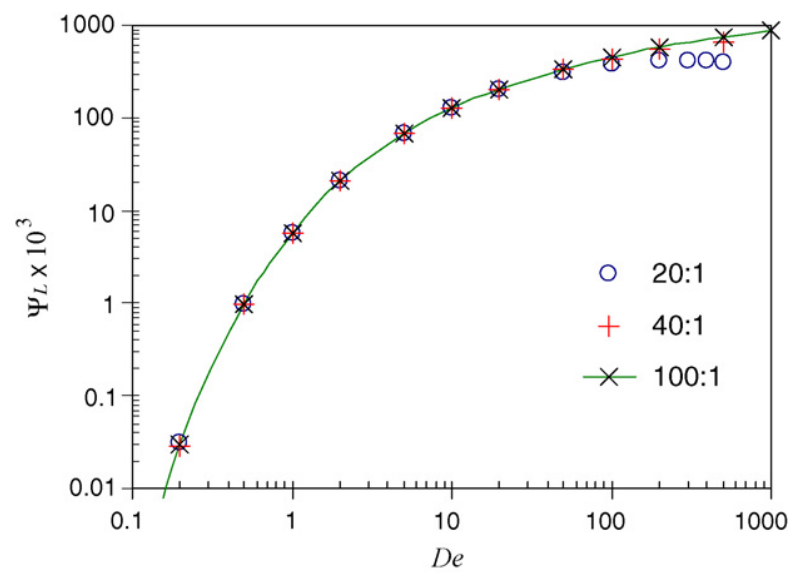

Fig. 15. Variation of lip vortex intensity with $D e$ for a PTT fluid $(\varepsilon=0.25$, $\beta=1 / 9$ ).

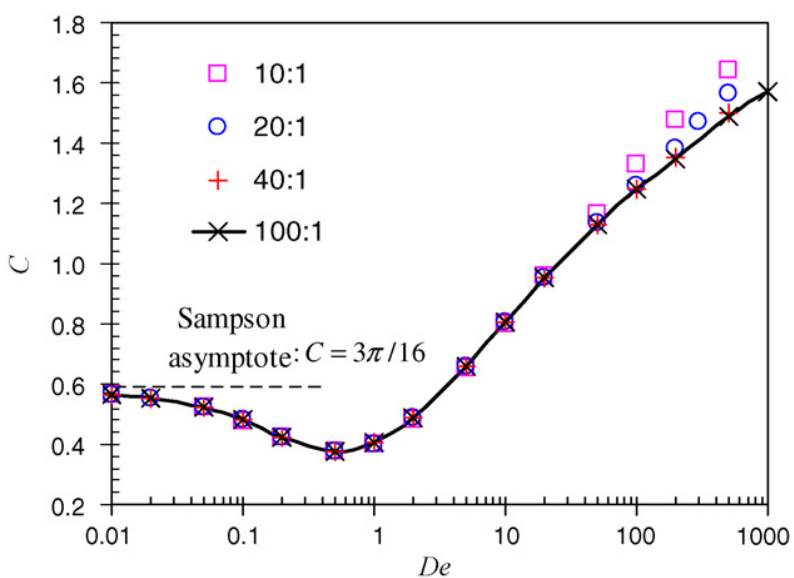

Fig. 16. Variation of Couette correction with $D e$ for a PTT fluid $(\varepsilon=0.25$, $\beta=1 / 9$ ).

Oldroyd-B model, the analysis is restricted to a much narrower range of Deborah numbers.

Figs. 17-19 show the effect of $\varepsilon$ on the size of the corner vortex, the intensity of the lip vortex and the Couette correction, respectively, for $\mathrm{CR}=20$. The variable used in the abscissa is the

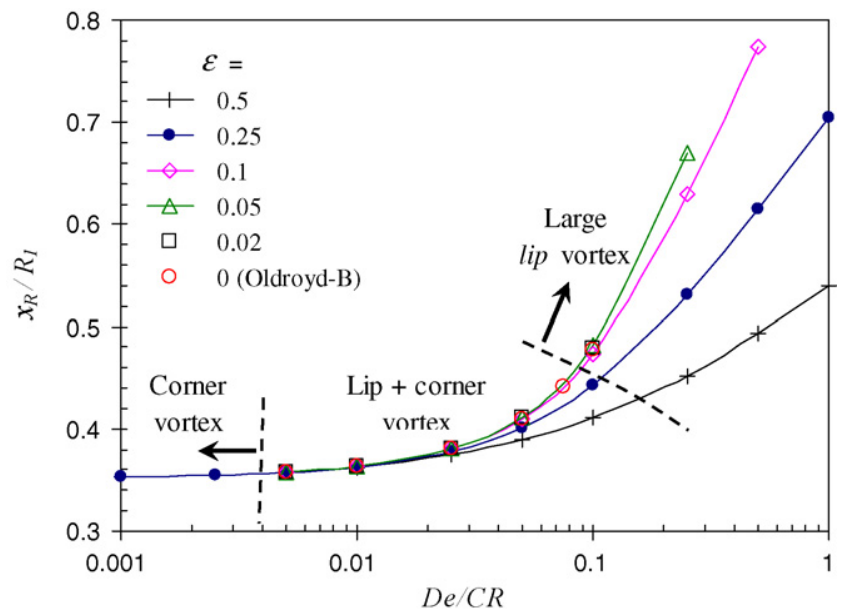

Fig. 17. Effect of the $\varepsilon$ parameter on the corner-vortex size $(C R=20)$.

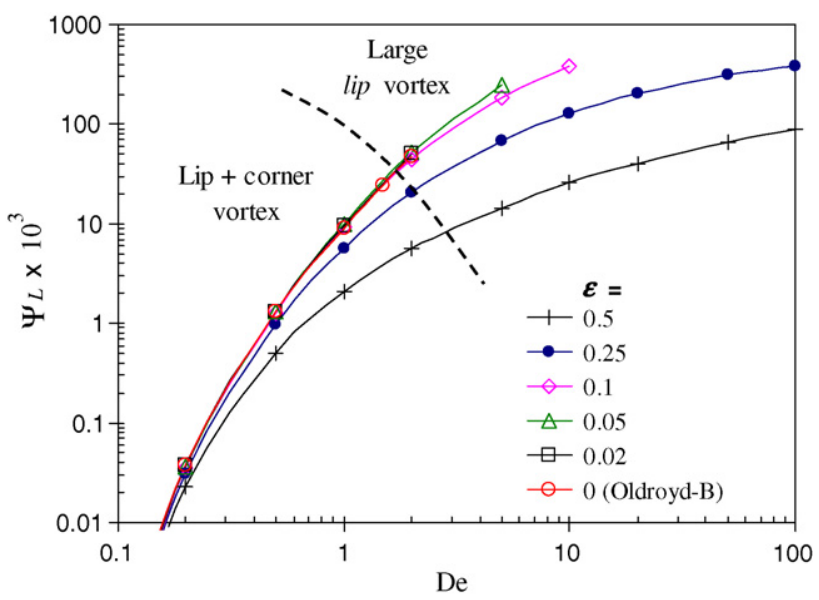

Fig. 18. Effect of the $\varepsilon$ parameter on the lip vortex intensity $(C R=20)$.

controlling parameter for each property ( $D e$ for the lip-vortex intensity and the Couette correction, and $D e / \mathrm{CR}$ for the cornervortex size).

For the range of low $D e$ (and $D e / \mathrm{CR}$ ), which corresponds to the corner-vortex region of the vortex map in Fig. 12(a), the results of Couette correction, vortex size and intensity are identical for the two constitutive models used. At these low values of $D e$, the non-linear term of the PTT model is small and the results are insensitive to the value of $\varepsilon$. However, for higher values of $D e$ (and $D e / \mathrm{CR}$ ), the results are highly influenced by $\varepsilon$. For instance, at high $D e / \mathrm{CR}$ when lip vortices are present, the size of the vortex that reaches the outer wall increases in inverse proportion to the value of $\varepsilon$ (cf. Fig. 17). Similarly, the evolution of the lip-vortex intensity with $D e$ depends strongly on the value of $\varepsilon$, showing intensification as $\varepsilon$ decreases (Fig. 18). At high $D e$, the magnitude of the Couette correction becomes also very sensitive to the $\varepsilon$ parameter (Fig. 19). For the linear PTT model with the standard parameters used in the previous section $(\varepsilon=0.25)$, the extra pressure loss due to the contraction entry leads to positive values of the Couette correction for the whole range of $D e$ tested, while it may reach negative values as $\varepsilon$ is decreased. For the particular case of the Oldroyd-B fluid $(\varepsilon=0)$, the Couette correction becomes negative for Deborah numbers

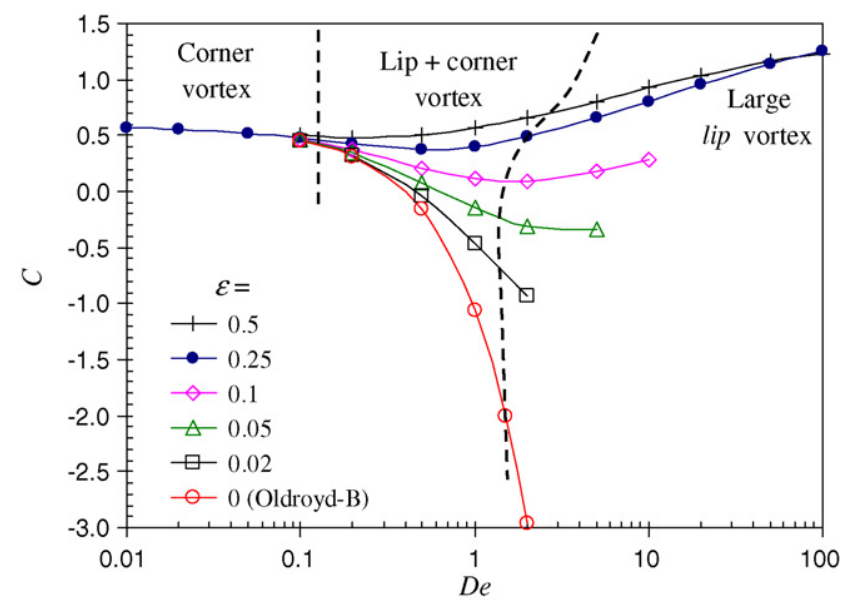

Fig. 19. Effect of the $\varepsilon$ parameter on the Couette correction $(C R=20)$. 


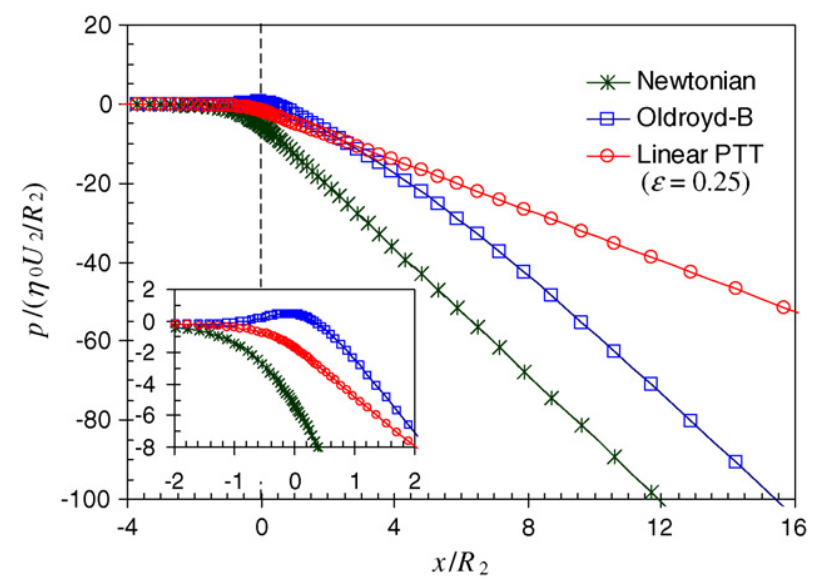

Fig. 20. Comparison of the pressure profiles along the $x$-axis for different rheological models $(\mathrm{CR}=40)$.

larger than 0.4. A negative value indicates a pressure gain as the fluid goes through the contraction $\left(\Delta P_{\text {total }}<\Delta P_{\text {Poiseuille }}\right)$, i.e. an elastic recovery. However, such an elastic recovery has not yet been observed in experiments, not even with Boger fluids, a finding that is corroborated by other numerical works (e.g. [37,68]). Based on previous experimental observations and numerical calculations, Rothstein and McKinley [10,20] suggested that the inability of the Oldroyd-B constitutive model to predict the correct variation of the pressure drop with the Deborah number may be related to an inadequate description of the internal molecular conformations of the polymer chains that arise during rapid stretching.

The pressure profiles along the pipe axis are shown in dimensionless form in Fig. 20 for a Newtonian, an Oldroyd-B and a standard $\mathrm{PTT}$ fluid, in the $\mathrm{CR}=40$ geometry. For the viscoelastic fluids the results are for $D e=2$. Examination of the region close to the contraction plane (dashed line represents $x=0$ ), there is a clear pressure overshoot for the Oldroyd-B fluid. Downstream of the contraction, the pressure corresponding to the Oldroyd-B model starts to decrease sharply with an increasing slope. Far downstream of the contraction $\left(x / R_{2} \approx 12\right)$, the slope approaches that of the Newtonian fluid (with the same viscosity). For the same Deborah number, the curve for the Oldroyd-B model is steeper than for the PTT fluid because of shear-thinning effects in the latter model.

The profiles of streamwise velocity and corresponding strainrate, along the centerline for the standard PTT fluid, are shown in Fig. 21( $\mathrm{a}$ and b), respectively. These were obtained for $\mathrm{CR}=40$ and Deborah numbers in the range $0.1 \leq D e \leq 500$. For comparison, the results for a Newtonian fluid are also included. For low $D e$, at a position far upstream of the contraction where the shear rate is low and where there are no shear-thinning or extensional effects, the centerline velocity is approximately twice the average velocity in the large pipe $\left(U_{1}\right)$, such as in Newtonian Poiseuille flow. As the fluid approaches the contraction, the velocity increases significantly when the diameter of the pipe suddenly decreases. When the Deborah number increases the dimensionless velocity gradient along the axis (i.e. the dimensionless strain-rate) is reduced and the entrance effects are felt
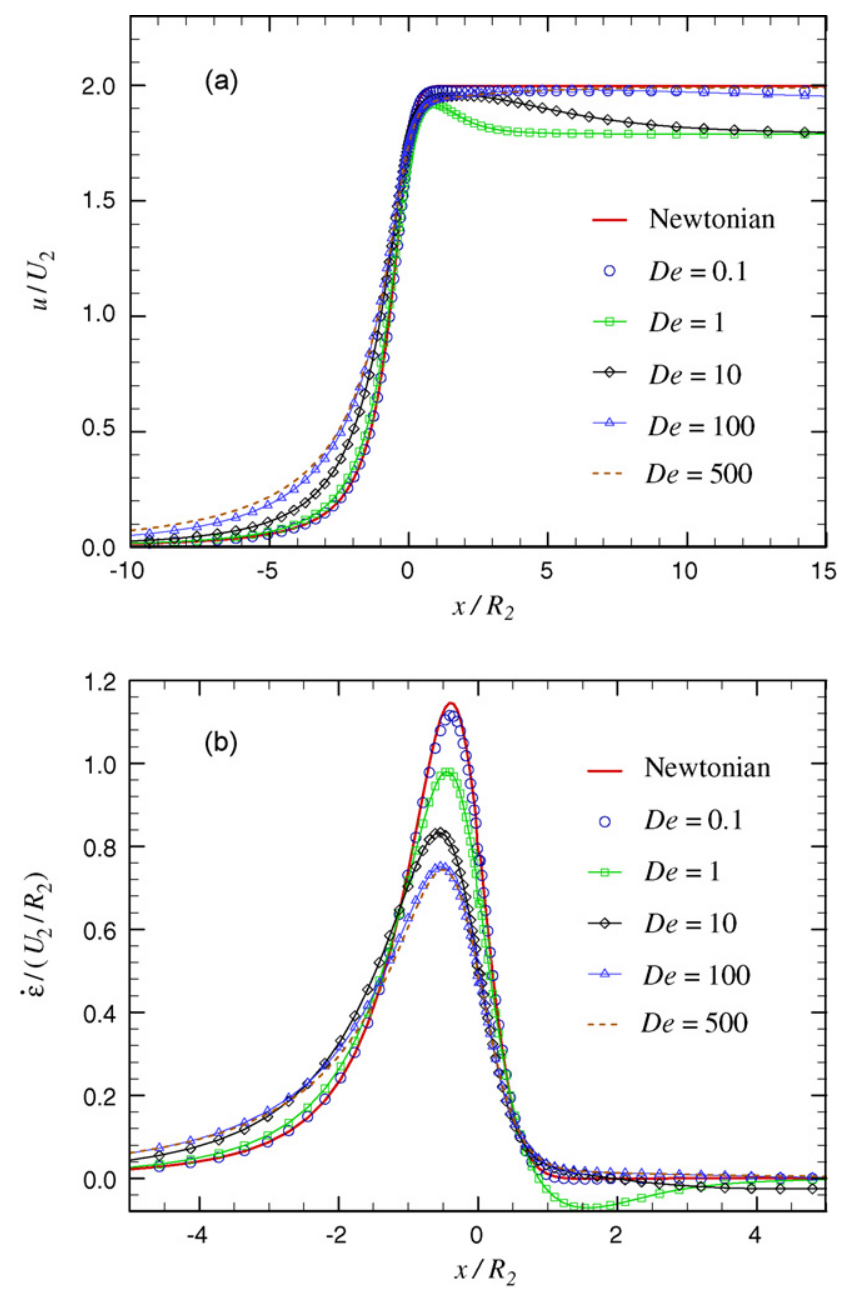

Fig. 21. Profiles along the $x$-axis for the standard PTT model $(\varepsilon=0.25, \beta=1 / 9)$ with $\mathrm{CR}=40$ : (a) velocity profiles and (b) strain-rate profiles.

further upstream of the contraction plane due to elastic effects. Downstream of the contraction plane the flow fully re-develops and, for low $D e$, the velocity profile of the PTT fluid considered here is identical to that of the Newtonian fluid. However, in an intermediate range of Deborah numbers $(D e=1$ and 10), there is a sharp velocity overshoot near the contraction plane, indicating a tendency for the elastic flow to converge towards the axis (very similar to what occurs in the reverse problem, of flow through expansions, see Oliveira [11] and Poole et al. [69]). Although the location of the maximum velocity does not change significantly, it takes longer for the stresses to relax for the higher $D e$ numbers. In these cases, the radial profiles of velocity are flatter and therefore the maximum velocity attained at the centerline is lower than the expected value of $2 U_{2}$ for Poiseuille flow of a Newtonian fluid on account of shear-thinning effects. At the largest Deborah number plotted $(D e=500)$, the fully developed velocity profile resembles once more that of a Newtonian fluid. At such high $D e$, the shear viscosity is significantly smaller than $\eta_{0}$ (approaching $\eta_{\mathrm{s}}$, here $\eta_{\mathrm{s}} / \eta_{0}=1 / 9$ ), and the Newtonian component of the shear stress becomes the dominant term. It should be emphasized that the extensional behavior of the Newtonian fluid and of the PTT fluid is significantly different since the PTT fluid 

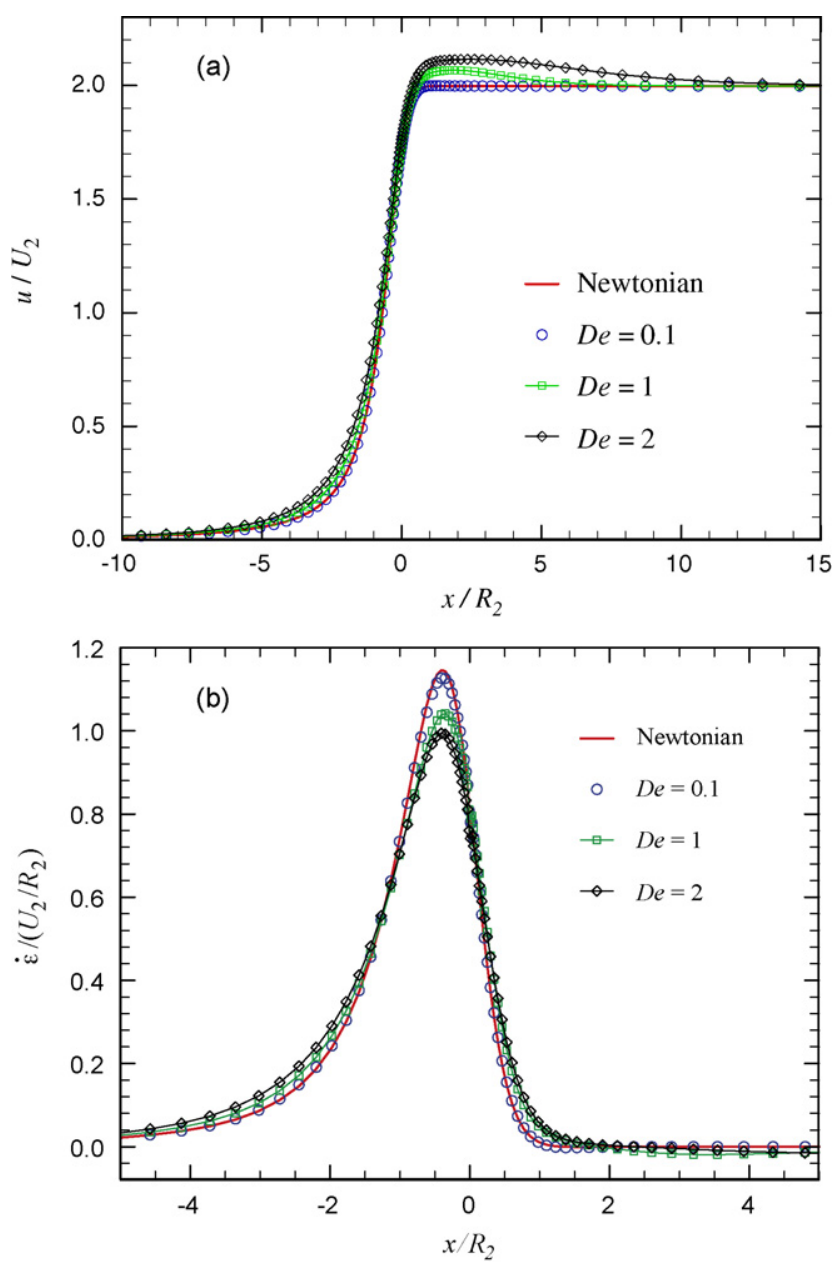

Fig. 22. Profiles along the $x$-axis for the Oldroyd-B $\operatorname{model}(\beta=1 / 9)$ and $C R=40$ : (a) velocity profiles and (b) strain-rate profiles.

is extensionally thickening until it reaches a plateau in extensional viscosity at high strain rates, whereas a Newtonian fluid always exhibits a constant extensional viscosity.

For the Oldroyd-B fluid (Fig. 22 for $\mathrm{CR}=40$ ), the velocity profiles upstream of the contraction are similar to those described above for the PTT fluid, except that now a velocity overshoot is also observed in the range of $D e$ obtainable. The magnitude of this overshoot increases with the Deborah number, and the fluid takes longer to fully re-develop, but as expected the velocity at the centerline downstream of the contraction reduces to the value for Newtonian Poiseuille flow further downstream of the contraction $\left(2 U_{2}\right)$.

\section{The normal-stress ratio criterion}

Rothstein and McKinley [10] showed that competing extensional stresses and shear-induced normal stresses play a decisive role on the pathway of vortex enhancement that a fluid will follow in a contraction-expansion flow. They define a dimensionless normal-stress ratio, $N$, and found that there is a critical value of $N \approx 0.055 \pm 0.005$ below which corner-vortex growth is observed and above which lip-vortex growth is observed. This normal-stress ratio is the ratio of the first normal-stress differ- ence in steady shear flow to that in transient uniaxial extension:

$N \equiv \frac{N_{1} / \eta_{0} \dot{\gamma}}{\left(\tau_{x x}-\tau_{r r}\right) / \eta_{0} \dot{\varepsilon}}=\frac{\operatorname{Sr}(\dot{\gamma})}{\operatorname{Tr}\left(\varepsilon_{\mathrm{H}}\right)}$

where $\operatorname{Tr}\left(\varepsilon_{\mathrm{H}}\right)$ is the Trouton ratio evaluated at the total Hencky strain accumulated along the axial distance, $\varepsilon_{\mathrm{H}}, \operatorname{Sr}(\dot{\gamma})$ the shearrate-dependent stress ratio and $\dot{\gamma}$ is the shear rate, which can be estimated as $\dot{\gamma} \approx \dot{\varepsilon}=U_{2} / R_{2}$ [10].

Following their approach, we plot the normal-stress ratio as a function of the Deborah number for different fluids and different contraction ratios (Fig. 23). Values of $N$ were obtained directly from the material functions and Eq. (10) without resorting to numerical simulations of the actual contraction flows. Subsequently, based on the results of the simulation, a closed or open symbol was given to each data point in Fig. 23 depending on the vortex pattern predicted numerically. The open symbols correspond to the salient-corner region and the closed symbols correspond to the region where corner and lip vortices are seen to co-exist, or where a large lip-vortex dominates. Also marked in the figure is the critical range of the normal-stress ratio $(N \approx 0.055 \pm 0.005)$, which Rothstein and McKinley [10] found to divide the data into two regions. Clearly, for the large contraction ratios, the normal-stress ratio can be used successfully as a method to predict the onset of lip-vortex activity. Hence, knowing the curve of $N$ versus $D e$ and using this criterion, one is able to identify if there is lip-vortex activity for a certain $D e$ and to predict the approximate value of $D e$ at which lip-vortex onset will occur, with the benefit of avoiding expensive numerical calculations. We note that the onset of lip-vortex activity coincides with the Deborah number for which the normal-stress ratio attains a value close to 0.055 , when approached from below. After a lip-vortex structure has been established, and $N=0.055$ is approached from above (as some of the data points shown by closed symbols below the line $N=0.055$ in Fig. 23 indicate), then no further change of vortex type should occur.

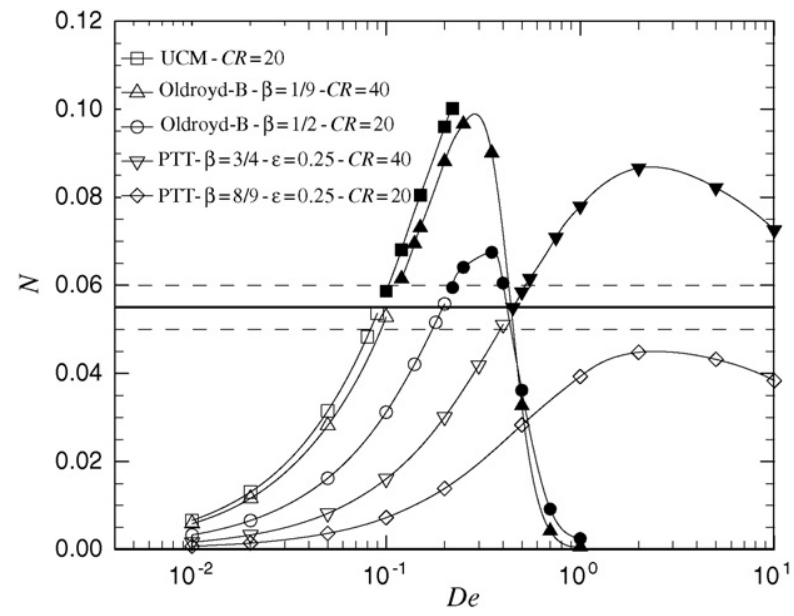

Fig. 23. Normal-stress ratio as a function of the Deborah number for different contraction ratios and rheological models. The open symbols correspond to corner-vortex region, and the closed symbols to the region where corner and lip vortices co-exist, or where a large lip vortex dominates. 


\section{Conclusions}

Comprehensive numerical simulations with a finite-volume method of PTT viscoelastic flow through axisymmetric abrupt contractions of varying contraction ratio, from 2 up to 100 , produced results for the vortex characteristics and the formation mechanism similar to those found in a previous study for the planar case [39]. The inception of a lip-vortex mechanism and its intensification are controlled by a Deborah number defined in the usual way, based on downstream quantities $\left(D e=\lambda U_{2} / R_{2}\right)$, starting at $D e \approx 0.1-0.2$ (compared with the range $1-2$ for the planar case). On the other hand, the characteristics of the corner vortices are controlled by a scaled Deborah number, $D e / C R$.

The Couette correction, a normalized measure of the local pressure loss due to the contraction, was seen to first decrease with $D e$ and then, for $D e>1$, to increase up to values three times greater than those obtained for a Newtonian fluid. This variation, valid for the PTT model with $\varepsilon=0.25$, was very sensitive to the extensibility parameter used in the PTT model. For low values of $\varepsilon$, and namely for $\varepsilon \rightarrow 0$ leading to the Oldroyd$\mathrm{B}$ model, the Couette correction never exceeds the Newtonian value (which is itself in excellent agreement with the theory of Sampson) and even becomes negative (elastic recovery) for the Oldroyd-B model when $D e$ increases, as also found by other authors.

The vortex type (corner, lip or mixed) is quantified in a twodimensional map with $\mathrm{CR}$ and $D e$ as independent parameters. A discerning criterion proposed by Rothstein and McKinley [10] in which the vortex type correlates with a normal-stress ratio of shear and elongational stresses, was shown to match reasonably well the results of our simulations. Finally, the paper also provides vortex data of benchmark quality for the 4:1 contraction test case, which may be useful for code assessment since the existing results in the literature exhibit too much scatter.

\section{Acknowledgements}

M.S.N. Oliveira wishes to thank Fundação para a Ciência e a Tecnologia (FCT) for financial support (SFRH/BPD/ 15005/2004 and SFRH/BPD/34141/2006). M.A. Alves acknowledges the financial support provided under program POCI2010 by FCT and FEDER through project POCI/EQU/ $59256 / 2004$.

\section{References}

[1] D.V. Boger, Viscoelastic flows through contractions, Annu. Rev. Fluid Mech. 19 (1987) 157-182.

[2] J. Kestin, M. Sokolov, W. Wakeham, Theory of capillary viscometers, Appl. Sci. Res. 27 (1973) 241-264.

[3] J.S. Vrentas, J.L. Duda, K.G. Bargeron, Effect of axial diffusion of vorticity on flow development in circular conduits. 1. Numerical solutions, AIChE J. 12 (1966) 837-844.

[4] H.A. Barnes, J.F. Hutton, K. Walters, An Introduction to Rheology, Elsevier: Distributors for the U.S. and Canada, Elsevier Science Pub. Co., Amsterdam/New York, 1989.

[5] S.A. White, A.D. Gotsis, D.G. Baird, Review of the entry flow problem-experimental and numerical, J. Non-Newtonian Fluid Mech. 24 (1987) 121-160.
[6] M.A. Alves, F.T. Pinho, P.J. Oliveira, Visualizations of Boger fluid flows in a 4:1 square-square contraction, AIChE J. 51 (2005) 2908-2922.

[7] L.E. Rodd, T.P. Scott, D.V. Boger, J.J. Cooper-White, G.H. McKinley, The inertio-elastic planar entry flow of low-viscosity elastic fluids in microfabricated geometries, J. Non-Newtonian Fluid Mech. 129 (2005) 1-22.

[8] K. Walters, M.F. Webster, The distinctive CFD challenges of computational rheology, Int. J. Numer. Methods Fluids 43 (2003) 577-596.

[9] R.G. Owens, T.N. Phillips, Computational Rheology, Imperial College Press, London, 2002.

[10] J.P. Rothstein, G.H. McKinley, The axisymmetric contraction-expansion: the role of extensional rheology on vortex growth dynamics and the enhanced pressure drop, J. Non-Newtonian Fluid Mech. 98 (2001) 33-63.

[11] P.J. Oliveira, Asymmetric flows of viscoelastic fluids in symmetric planar expansion geometries, J. Non-Newtonian Fluid Mech. 114 (2003) 33-63.

[12] P.J. Cable, D.V. Boger, Comprehensive experimental investigation of tubular entry flow of viscoelastic fluids. 1. Vortex characteristics in stable flow, AIChE J. 24 (1978) 869-879.

[13] P.J. Cable, D.V. Boger, Comprehensive experimental investigation of tubular entry flow of viscoelastic fluids. 2. Velocity-field in stable flow, AIChE J. 24 (1978) 992-999.

[14] P.J. Cable, D.V. Boger, Comprehensive experimental investigation of tubular entry flow of viscoelastic fluids. 3. Unstable flow, AIChE J. 25 (1979) $152-159$.

[15] H. Nguyen, D.V. Boger, Kinematics and stability of die entry flows, J. Non-Newtonian Fluid Mech. 5 (1979) 353-368.

[16] D.V. Boger, D.U. Hur, R.J. Binnington, Further observations of elastic effects in tubular entry flows, J. Non-Newtonian Fluid Mech. 20 (1986) 31-49.

[17] G.H. McKinley, W.P. Raiford, R.A. Brown, R.C. Armstrong, Nonlinear dynamics of viscoelastic flow in axisymmetrical abrupt contractions, J. Fluid Mech. 223 (1991) 411-456.

[18] D.V. Boger, R.J. Binnington, Experimental removal of the reentrant corner singularity in tubular entry flows, J. Rheol. 38 (1994) 333-349.

[19] J.M. Maia, D. Binding, Influence of elongational properties on the contraction flow of polyisobutylene in a mixed solvent, Rheol. Acta 38 (1999) $160-171$.

[20] J.P. Rothstein, G.H. McKinley, Extensional flow of a polystyrene Boger fluid through a 4:1:4 axisymmetric contraction/expansion, J. NonNewtonian Fluid Mech. 86 (1999) 61-88.

[21] M.J. Crochet, A.R. Davies, K. Walters, Numerical Simulation of nonNewtonian Flow, Elsevier, Amsterdam/New York, 1984.

[22] S.A. White, D.G. Baird, The importance of extensional flow properties on planar entry flow patterns of polymer melts, J. Non-Newtonian Fluid Mech. 20 (1986) 93-101.

[23] S.A. White, D.G. Baird, Flow visualization and birefringence studies on planar entry flow behavior of polymer melts, J. Non-Newtonian Fluid Mech. 29 (1988) 245-267.

[24] S.A. White, D.G. Baird, Numerical-simulation studies of the planar entry flow of polymer melts, J. Non-Newtonian Fluid Mech. 30 (1988) $47-71$.

[25] R.E. Evans, K. Walters, Flow characteristics associated with abrupt changes in geometry in the case of highly elastic liquids, J. Non-Newtonian Fluid Mech. 20 (1986) 11-29.

[26] R.E. Evans, K. Walters, Further remarks on the lip-vortex mechanism of vortex enhancement in planar-contraction flows, J. Non-Newtonian Fluid Mech. 32 (1989) 95-105.

[27] K. Chiba, T. Sakatani, K. Nakamura, Anomalous flow patterns in viscoelastic entry flow through a planar contraction, J. Non-Newtonian Fluid Mech. 36 (1990) 193-203.

[28] K. Chiba, S. Tanaka, K. Nakamura, The structure of anomalous entry flow patterns through a planar contraction, J. Non-Newtonian Fluid Mech. 42 (1992) 315-322.

[29] L.M. Quinzani, R.C. Armstrong, R.A. Brown, Use of coupled birefringence and LDV studies of flow-through a planar contraction to test constitutive-equations for concentrated polymer-solutions, J. Rheol. 39 (1995) 1201-1228.

[30] J.M. Marchal, M.J. Crochet, A new mixed finite-element for calculating viscoelastic flow, J. Non-Newtonian Fluid Mech. 26 (1987) 77-114. 
[31] A. Baloch, P. Townsend, M.F. Webster, On the simulation of highly elastic complex flows, J. Non-Newtonian Fluid Mech. 59 (1995) 111128.

[32] H. Matallah, P. Townsend, M.F. Webster, Recovery and stress-splitting schemes for viscoelastic flows, J. Non-Newtonian Fluid Mech. 75 (1998) $139-166$.

[33] S.C. Xue, N. Phan-Thien, R.I. Tanner, Three dimensional numerical simulations of viscoelastic flows through planar contractions, J. Non-Newtonian Fluid Mech. 74 (1998) 195-245.

[34] P.J. Oliveira, F.T. Pinho, Plane contraction flows of upper convected Maxwell and Phan-Thien-Tanner fluids as predicted by a finite-volume method, J. Non-Newtonian Fluid Mech. 88 (1999) $63-$ 88.

[35] M.A. Alves, F.T. Pinho, P.J. Oliveira, Effect of a high-resolution differencing scheme on finite-volume predictions of viscoelastic flows, J. Non-Newtonian Fluid Mech. 93 (2000) 287-314.

[36] M. Aboubacar, M.F. Webster, A cell-vertex finite volume/element method on triangles for abrupt contraction viscoelastic flows, J. Non-Newtonian Fluid Mech. 98 (2001) 83-106.

[37] M. Aboubacar, H. Matallah, H.R. Tamaddon-Jahromi, M.F. Webster, Numerical prediction of extensional flows in contraction geometries: hybrid finite volume/element method, J. Non-Newtonian Fluid Mech. 104 (2002) 125-164.

[38] M.A. Alves, P.J. Oliveira, F.T. Pinho, Benchmark solutions for the flow of Oldroyd-B and PTT fluids in planar contractions, J. Non-Newtonian Fluid Mech. 110 (2003) 45-75.

[39] M.A. Alves, P.J. Oliveira, F.T. Pinho, On the effect of contraction ratio in viscoelastic flow through abrupt contractions, J. Non-Newtonian Fluid Mech. 122 (2004) 117-130.

[40] O. Hassager, Working group on numerical techniques (Vth Workshop on Numerical Methods in Non-Newtonian Flow), J. Non-Newtonian Fluid Mech. 29 (1988) 2-5.

[41] K. Walters, M.F. Webster, On dominating elastico-viscous response in some complex flows, Philos. Trans. R. Soc. London Ser. A-Math. Phys. Eng. Sci. 308 (1982) 199-218

[42] K. Walters, D.M. Rawlinson, On some contraction flows for Boger fluids, Rheol. Acta 21 (1982) 547-552.

[43] S.C. Xue, N. Phan-Thien, R.I. Tanner, Numerical investigations of Lagrangian unsteady extensional flows of viscoelastic fluids in 3-D rectangular ducts with sudden contractions, Rheol. Acta 37 (1998) 158169.

[44] P.J. Oliveira, F.T. Pinho, Pressure drop coefficient of laminar Newtonian flow in axisymmetric sudden expansions, Int. J. Heat Fluid Flow 18 (1997) $518-529$.

[45] S. Sisavath, X.D. Jing, C.C. Pain, R.W. Zimmerman, Creeping flow through an axisymmetric sudden contraction or expansion, J. Fluids Eng. Trans. ASME 124 (2002) 273-278.

[46] F.N. Cogswell, Converging flow of polymer melts in extrusion dies, Polym. Eng. Sci. 12 (1972) 64-73.

[47] D.M. Binding, M.A. Couch, K. Walters, The pressure dependence of the shear and elongational properties of polymer melts, J. Non-Newtonian Fluid Mech. 79 (1998) 137-155.

[48] D.V. Boger, R.J. Binnington, Circular entry flows of fluid M1, J. NonNewtonian Fluid Mech. 35 (1990) 339-360.

[49] D.F. James, G.M. Chandler, S.J. Armour, A converging channel rheometer for the measurement of extensional viscosity, J. Non-Newtonian Fluid Mech. 35 (1990) 421-443.
[50] M.S.N. Oliveira, M.A. Alves, F.T. Pinho, G.H. McKinley, Viscous flow through microfabricated hyperbolic contractions, Exp. Fluids 43 (2007) 437-451.

[51] M.S.N. Oliveira, L.E. Rodd, G.H. McKinley, M.A. Alves, Simulations of extensional flow in microrheometric devices, Microfluid. Nanofluid. submitted for publication.

[52] S. Nigen, K. Walters, Viscoelastic contraction flows: comparison of axisymmetric and planar configurations, J. Non-Newtonian Fluid Mech. 102 (2002) 343-359.

[53] R. Keunings, M.J. Crochet, Numerical-simulation of the flow of a viscoelastic fluid through an abrupt contraction, J. Non-Newtonian Fluid Mech. 14 (1984) 279-299.

[54] S.D. Phillips, Y.L. Joo, R.A. Brown, R.C. Armstrong, Modeling and simulation of the flow through an axisymmetric contraction-expansion using a multi-mode adaptive length scale model, Paper NF25, in: Proc. of the XIV International Congress on Rheology, Seoul, Korea, 2004.

[55] I. Ghosh, Y.L. Joo, G.H. McKinley, R.A. Brown, R.C. Armstrong, A new model for dilute polymer solutions in flows with strong extensional components, J. Rheol. 46 (2002) 1057-1089.

[56] S. Meng, X.K. Li, G. Evans, Spectral element method for viscoelastic flows in a planar contraction channel, Int. J. Numer. Methods Fluids 42 (2003) 323-348.

[57] S.S. Edussuriya, A.J. Williams, C. Bailey, A cell-centred finite volume method for modelling viscoelastic flow, J. Non-Newtonian Fluid Mech. 117 (2004) 47-61.

[58] D. Trebotich, P. Colella, G.H. Miller, A stable and convergent scheme for viscoelastic flow in contraction channels, J. Comput. Phys. 205 (2005) 315-342.

[59] J.M. Kim, C. Kim, J.H. Kim, C. Chung, K.H. Ahn, S.J. Lee, High-resolution finite element simulation of 4:1 planar contraction flow of viscoelastic fluid, J. Non-Newtonian Fluid Mech. 129 (2005) 23-37.

[60] N. Phan-Thien, R.I. Tanner, New constitutive equation derived from network theory, J. Non-Newtonian Fluid Mech. 2 (1977) 353-365.

[61] P.J. Oliveira, F.T. Pinho, G.A. Pinto, Numerical simulation of non-linear elastic flows with a general collocated finite-volume method, J. NonNewtonian Fluid Mech. 79 (1998) 1-43.

[62] M.A. Alves, P.J. Oliveira, F.T. Pinho, A convergent and universally bounded interpolation scheme for the treatment of advection, Int. J. Numer. Methods Fluids 41 (2003) 47-75.

[63] B.P. Leonard, Stable and accurate convective modeling procedure based on quadratic upstream interpolation, Comput. Methods Appl. Mech. Eng. 19 (1979) 59-98.

[64] T.N. Phillips, A.J. Williams, Comparison of creeping and inertial flow of an Oldroyd B fluid through planar and axisymmetric contractions, J. NonNewtonian Fluid Mech. 108 (2002) 25-47.

[65] D.V. Boger, K. Walters, Rheological Phenomena in Focus, Elsevier, Amsterdam/New York, 1993.

[66] H.K. Moffatt, Viscous and resistive eddies near a sharp corner, J. Fluid Mech. 18 (1964) 1-18.

[67] M.A. Alves, R.J. Poole, Divergent flow in contractions, J. Non-Newtonian Fluid Mech. 144 (2007) 140-148.

[68] P.J. Coates, R.C. Armstrong, R.A. Brown, Calculation of steady-state viscoelastic flow through axisymmetrical contractions with the EEME formulation, J. Non-Newtonian Fluid Mech. 42 (1992) 141-188.

[69] R.J. Poole, M.A. Alves, P.J. Oliveira, F.T. Pinho, Plane sudden expansion flows of viscoelastic liquids, J. Non-Newtonian Fluid Mech. 146 (2007) 79-91. 